\title{
Host Plant Adaptation Drives Changes in Diaphorina citri Proteome Regulation, Proteoform Expression, and Transmission of 'Candidatus Liberibacter asiaticus', the Citrus Greening Pathogen
}

\author{
John S. Ramsey, ${ }^{1}$ El-Desouky Ammar, ${ }^{2}$ Jaclyn E. Mahoney, ${ }_{5}^{3}$ Keith Rivera, ${ }^{4}$ Richard Johnson, ${ }^{5}$ David O. Igwe, ${ }^{6}$ \\ Theodore W. Thannhauser, ${ }^{7}$ Michael J. MacCoss, ${ }^{5}$ David G. Hall, ${ }^{2}$ and Michelle Heck ${ }^{1,6, \dagger}$ \\ ${ }^{1}$ U.S. Department of Agriculture-Agricultural Research Service-Emerging Pests and Pathogens Research Unit, Ithaca, NY \\ ${ }^{2}$ U.S. Department of Agriculture-Agricultural Research Service, USHRL-SIRU, Fort Pierce, FL \\ ${ }^{3}$ Boyce Thompson Institute for Plant Research, Ithaca, NY \\ ${ }^{4}$ Cold Spring Harbor Laboratory, Cold Spring Harbor, NY \\ ${ }^{5}$ University of Washington-Genome Sciences, Seattle, WA \\ ${ }^{6}$ Cornell University College of Agriculture and Life Sciences-Plant Pathology and Plant Microbe Biology, Ithaca, NY \\ ${ }^{7}$ U.S. Department of Agriculture-Agricultural Research Service-Plant, Soil, and Nutrition Research Unit, Ithaca, NY \\ Accepted for publication 22 September 2021.
}

\begin{abstract}
The Asian citrus psyllid (Diaphorina citri) is a pest of citrus and the primary insect vector of the bacterial pathogen, 'Candidatus Liberibacter asiaticus' (CLas), which is associated with citrus greening disease. The citrus relative Murraya paniculata (orange jasmine) is a host plant of $D$. citri but is more resistant to CLas compared with all tested Citrus genotypes. The effect of host switching of D. citri between Citrus medica (citron) and M. paniculata plants on the acquisition and transmission of $C$ Las was investigated. The psyllid $C$ Las titer and the proportion of $C$ Lasinfected psyllids decreased in the generations after transfer from CLasinfected citron to healthy M. paniculata plants. Furthermore, after several

continually maintained on infected citron. Top-down (difference gel electrophoresis) and bottom-up (shotgun MS/MS) proteomics methods were used to identify changes in $D$. citri protein expression resulting from host plant switching between Citrus macrophylla and M. paniculata. Changes in expression of insect metabolism, immunity, and cytoskeleton proteins were associated with host plant switching. Both transient and sustained feeding on $M$. paniculata induced distinct patterns of protein expression in D. citri compared with psyllids reared on $C$. macrophylla. The results point to complex interactions that affect vector competence and may lead to strategies to control the spread of citrus greening disease.
\end{abstract} generations of feeding on M. paniculata, pathogen acquisition (20 to 40\% reduction) and transmission rates (15 to $20 \%$ reduction) in psyllids transferred to CLas-infected citron were reduced compared with psyllids
Keywords: bacterial pathogens, proteomics, systems biology
Insect pests transmit innumerable pathogens to humans, livestock, and crop plants, representing a worldwide threat to public health and agricultural production (Heck 2018). Vector competence depends on a complex set of interacting factors, including environmental factors as well as the genetics of the insect, pathogen, and plant or animal host (Beerntsen et al. 2000; Heck 2018). The Asian citrus psyllid (Diaphorina citri, Hemiptera: Liviidae) is an insect vector of 'Candidatus Liberibacter asiaticus' (CLas), a bacterial pathogen associated with huanglongbing (HLB, citrus greening disease), and it has a broad host range, including citrus and non-citrus species within the family Rutaceae (Alves et al. 2021; Killiny 2022). Although no commercial citrus varieties are resistant to CLas, variation in CLas susceptibility has been reported among different citrus genotypes (McCollum et al. 2016), and resistant non-citrus wild relatives within Rutaceae have been identified (Ramadugu et al. 2016). Two citrus species commonly used for laboratory rearing of

${ }^{\dagger}$ Corresponding author: M. Heck; mlc68@ cornell.edu

Funding: This work was supported by Agricultural Research Service (grant 8062-22410-007-000-D), National Institute of Food and Agriculture (grant 201670016-24779), and California Citrus Research Board (grant 5300-163).

*The $\boldsymbol{e}$-Xtra logo stands for "electronic extra" and indicates that nine supplementary tables and two supplementary figures are published online.

The author(s) declare no conflict of interest.

This article is in the public domain and not copyrightable. It may be freely reprinted with customary crediting of the source. The American Phytopathological Society, 2022
D. citri are Citrus medica (citron) and Citrus macrophylla. C. macrophylla produces large amounts of flush tissue following pruning, which supports oviposition and nymphal development (Hall and Hentz 2016), and is highly susceptible to CLas, with pathogen titer increasing rapidly upon infection compared with other citrus species (McCollum et al. 2016). Citrus medica is a citrus host plant that is susceptible to CLas. It supports $D$. citri reproduction and development and has been used in detached-leaf $C$ Las transmission assays for HLB research (Ammar et al. 2013, 2018; Hall and Moulton 2018). The citrus relative Murraya paniculata is commonly grown as an ornamental hedge plant in citrus-growing regions and is a host for both $D$. citri and CLas (Cifuentes-Arenas et al. 2019; Damsteegt et al. 2010). Although M. paniculata is an excellent host of the insect because it continuously produces flush tissue, CLasinfected plants of this species are not commonly found in the field (Walter et al. 2012a). CLas titer was lower in M. paniculata than in commercial varieties of sweet orange (Citrus sinensis), and CLas titer was lower in D. citri reared on infected $M$. paniculata than in D. citri reared on $C$. sinensis (Walter et al. 2012b). The results of a recent study have shown that another non-citrus $D$. citri host species, Murraya koenigii, was even more resistant to CLas infection (Beloti et al. 2018).

Psyllid host plant experience is hypothesized to influence acquisition and transmission of CLas, and psyllids feeding on different host plants are hypothesized to have differences in protein expression. Host plant adaptation by $D$. citri has been shown to affect oviposition rates, development time (George and Lapointe 2018), and morphological traits such as size and wing shape (Paris et al. 2016). D. citri reared on Poncirus trifoliata, a rootstock commonly used in citrus 
production, has significantly reduced oviposition and nymphal infestation compared with colonies reared on C. sinensis (Hall et al. 2015). Analysis of morphometric variation between $D$. citri reared on different host plant species revealed statistically significant differences in wing shape: $D$. citri reared on $M$. paniculata had significantly narrower wings than isogenic insects reared on C. macrophylla (Paris et al. 2016). This variation in wing shape can impact dispersal behavior, with potential consequences on the effectiveness of the insect to serve as a pathogen vector. Insect wing shape variation may be associated with changes in flight muscle proteins or other components of the cytoskeleton (Hedrick et al. 2015).

Myzus persicae, the green peach aphid, was found to transmit Potato leafroll virus at higher rates when the insect was reared on Physalis floridana compared with turnip (Brassica rapa) (Pinheiro et al. 2017). Difference gel electrophoresis (DIGE) was used to compare the proteomes of aphids collected from each host plant, and a series of cathepsin B proteoforms was identified as overexpressed in turnip-reared compared with Physalis-reared M. persicae (Pinheiro et al. 2017). The functional significance of these proteases in plant virus transmission was revealed by artificial diet studies using the E64 cysteine protease inhibitor to block cathepsin B function, which restored the ability of turnip-reared aphids to transmit Potato leafroll virus.

The objective of this study was to evaluate the impact of $D$. citri host plant switching between Citrus and Murraya on the acquisition and transmission of $C$ Las and on the proteome profile of the insect vector.

\section{MATERIALS AND METHODS}

CLas quantitative PCR analysis. For these experiments, we used a colony of $D$. citri derived from insects collected from a citrus grove near Ft. Pierce, Florida, that were maintained on CLasinfected citron plants $(C$. medica) for several generations (at the U.S. Department of Agriculture lab in Fort Pierce, FL). Monthly CLas quantitative PCR (qPCR) tests were performed on colony insects to determine percent infection and average $C$ Las cycle threshold $(\mathrm{Ct})$ values. Insects collected from a healthy (CLas-unexposed) colony of D. citri, maintained on healthy Murraya plants, were used as negative controls in each qPCR test. Negative control sample $\mathrm{Ct}$ values were determined to be $\geq 40$.

DNA was isolated from insect and plant samples for $C$ Las qPCR analysis (Ammar et al. 2013). Individual adult insects were homogenized (30 s at $6 \mathrm{~m} / \mathrm{s}$, FastPrep 24 Homogenizer, MP Biomedicals, Inc.) in 1.5-ml microcentrifuge tubes containing $150 \mu \mathrm{l}$ of lysis buffer $(5 \% 1 \mathrm{M}$ of $\mathrm{KCl}, 5 \% 1 \mathrm{M}$ of Tris $\mathrm{pH} 8.4,0.45 \%$ Tween 20 , $0.45 \%$ NP-40, $89.1 \%$ autoclaved deionized water). Then $100 \mu \mathrm{l}$ of homogenate was transferred to a new $1.5-\mathrm{ml}$ microcentrifuge tube and incubated in a $95^{\circ} \mathrm{C}$ water bath for $5 \mathrm{~min}$. Homogenate was cooled on ice for $10 \mathrm{~min}$ and centrifuged at $14,000 \times g$ for $2 \mathrm{~min}$, and $2 \mu \mathrm{l}$ of the insect DNA supernatant was used as template for $C$ Las qPCR. For CLas analysis in plant samples, midribs were isolated from leaves and chopped into small pieces. Genomic DNA was isolated using the Machery Nagel Plant Kit II; $100 \mathrm{ng}$ of plant DNA was used as template for CLas qPCR.

The HLBaspr probe/primer set (Integrated DNA Technologies, Inc.) targeting CLas 16S DNA was used for $C$ Las qPCR analysis. Forward primer: TCGAGCGCGTATGCAATACG; reverse primer: GCGTTATCCCGTAGAAAAAGGTAG; probe: AGACGGGTGA GTAACGCG, with 6-carboxyflourescein reporter dye on the 5' end and TAMRA quencher on the $3^{\prime}$ end. A $20-\mu \mathrm{l}$ qPCR reaction was run using the Taqman Fast Universal PCR Master Mix (Applied Biosystems) using $0.4 \mathrm{mM}$ forward and reverse primer and 500-nM probe. qPCR reactions were run at $95^{\circ} \mathrm{C}$ for $5 \mathrm{~min}$, followed by 50 cycles $\left(95^{\circ} \mathrm{C}\right.$ for $3 \mathrm{~s}, 60^{\circ} \mathrm{C}$ for $\left.30 \mathrm{~s}\right)$.

D. citri host plant switching. Three host switch experiments were conducted as follows, with positive controls from the original $D$. citri colony maintained on infected citron for the duration of the experiment.
In host switch 1 (24 April 2017), two replicate groups of adult psyllids from the positive control $D$. citri colony reared on $C$ Las-infected C. medica (designated here as A and B with 100 adults in each group), were transferred to two different CLas-free $M$. paniculata plants confined in BugDorm muslin cages (BugDorm.com). qPCR tests were performed on adults from the progeny of these psyllids on 1 June 2017, 11 July 2017, and 17 August 2017, after two, four, and five/six generations on Murraya, respectively, considering that one generation of $D$. citri is estimated to take approximately 3 weeks under our rearing conditions $\left(25^{\circ} \mathrm{C}\right.$ and average relative humidity of 76\%) (Ammar et al. 2018; Hall et al. 2013).

In host switch $2(10 / 24 / 2017)$, after 6 months of rearing the above psyllids on healthy Murraya plants, two groups of $D$. citri adults (100 each) were collected from Murraya cages A and B and transferred to two new cages containing CLas-infected citron plants (with $\mathrm{Ct}$ value of 25 in qPCR test), also confined in BugDorm cages. Two qPCR tests, on 1 December 2017 and 2 February 2018, were performed on A and B adults (100/group/test) from the progeny of the transferred psyllids.

In host switch 3 (8 May 2018), after 12 months of rearing D. citri on healthy Murraya plants, 100 adults were collected from each of the two Murraya cages A and B and transferred to two new cages containing CLas-infected citron plants (with Ct value of 20 to 25 in qPCR tests). For statistical analysis, $\chi^{2}$ tests were performed on the proportion of infected (qPCR positive) psyllids or plants, and analysis of variance (ANOVA) tests were performed on the differences in $\mathrm{Ct}$ values between various groups/treatments.

CLas transmission assay. Adult psyllids were assayed for CLas transmission on excised healthy citron leaves (10 adults/leaf) (Ammar et al. 2013). At the end of the inoculation access period (7 days), live psyllids were removed from the leaves and stored individually at $-20^{\circ} \mathrm{C}$ and tested for CLas by qPCR. The inoculated excised leaves were incubated for 7 more days at $25^{\circ} \mathrm{C}$ before being tested for $C$ Las with qPCR.

D. citri rearing conditions for proteomics analysis. To test the hypothesis that host switching changes $D$. citri physiology and understand the molecular basis for a change in CLas transmission phenotype as a result of host switching, we performed a series of replicated host switch experiments and collected the insects for proteome analysis. Psyllids used in the proteomics studies were collected from colonies on CLas-free citrus plants and had no exposure to CLas. D. citri insects used in proteomics studies were lab reared under one of four host plant conditions: (i) psyllids were reared on M. paniculata for at least three generations (sample class: Murraya); (ii) psyllids were reared on C. macrophylla for at least three generations (sample class: $\mathrm{Cmac}$ ); (iii) psyllids were transferred to M. paniculata for 5 days after continuous rearing on C. macrophylla (sample class: Murraya host switch); and (iv) psyllids were transferred to $C$. macrophylla for 5 days after continuous rearing on $M$. paniculata (sample class: Cmac host switch). Colonies were derived from psyllids originally collected from an orange grove near Ft. Pierce, Florida. Colonies were maintained with a photoperiod of $14 \mathrm{~h}$ light and $10 \mathrm{~h}$ dark at $25^{\circ} \mathrm{C}$, and psyllids were flash frozen using liquid nitrogen after collection.

2D-DIGE sample preparation. Three biological replicates each of adult D. citri from the Murraya (100 insects each), Cmac (200 insects each), and Murraya host switch (200 insects each) sample classes were ground to fine powder using mortar, pestle, and liquid nitrogen. Proteins were extracted using a trichloroacetic acidacetone method as previously described (Cilia et al. 2009). Protein pellets were solubilized in rehydration buffer ( $7 \mathrm{M}$ of urea, $2 \mathrm{M}$ of thiourea, $4 \%$ CHAPS) overnight at room temperature. The supernatant was collected after centrifugation, and protein concentration was determined using the Quick Start Bradford Protein Assay (Bio-Rad).

Protein samples were labeled with cyanine dyes (CyDyes) $\mathrm{Cy} 3$ or Cy5 according to the manufacturer's instructions (GE Healthcare). A combined internal standard containing equal amounts of protein from 
each sample was labeled with $\mathrm{Cy} 2$ and used for relative quantification. A dye swap design was used to account for labeling bias; each sample was labeled with each dye once, and each gel contained a different comparison of samples in addition to the Cy2 combined internal standard. The dye/protein ratio for the experiments was $200 \mathrm{pmol}$ of dye $/ 50 \mu \mathrm{g}$ of protein. Analytical gels used for quantitative analysis contained a total of $150 \mu \mathrm{g}$ of protein: $50 \mu \mathrm{g}$ of CyDye labeled protein sample. Preparative gels used for spot picking contained a total of $750 \mu \mathrm{g}$ of nonlabeled protein. Samples were loaded onto Immobiline DryStrips (pH 4 to 7, $18 \mathrm{~cm}$, GE Healthcare) and allowed to passively rehydrate overnight according to the manufacturer's instructions. The first dimension was run on an IPGphor II Isoelectric Focusing System (GE Healthcare) according to the manufacturer's instruction for 18-cm Immobiline DryStrip gels of $\mathrm{pH} 4$ to 7, for a total of 28,000 VHrsT (Cilia et al. 2011b).

Gel strips were reduced and then alkylated using equilibration buffer (50 mM of Tris- $\mathrm{HCl}$ [pH 8.8], $6 \mathrm{M}$ of urea, $30 \%$ glycerol, $2 \%$ SDS, $0.002 \%$ bromophenol blue) with $64.8 \mathrm{mM}$ of dithiothreitol or $135.2 \mathrm{mM}$ of iodoacetamide added, respectively. The second dimension was performed with the Ettan Dalt 6 system using 8 to $15 \%$ gradient tris-glycine gels (Jule Biotechnology Inc.). Gels were run overnight at $25^{\circ} \mathrm{C}$ for a total of $19 \mathrm{~h}$ with the following steps: step 1, $10 \mathrm{~mA} / \mathrm{gel}$; step 2, $40 \mathrm{~mA} / \mathrm{gel}$.

Preparative gels were fixed using a solution of $10 \%$ methanol and $7 \%$ acetic acid for $30 \mathrm{~min}$ and then were stained overnight with Invitrogen NOVEX Colloidal Blue Staining Kit, following instructions for Tris-Glycine gels (Life Technologies) and destained in water for $24 \mathrm{~h}$ before spot picking for in-gel digestion and mass spectrometry.

Gel analysis. CyDye-labeled two-dimensional (2-D) gels were scanned on the Typhoon Variable Mode Imager Model 9400 (GE Healthcare) according to the manufacturer's specifications for CyDyes, and scan settings were optimized to achieve equal intensities within the linear dynamic range for the brightest protein spots on all three channels for each gel. Gel scans were analyzed using Progenesis SameSpots software (Nonlinear Dynamics). All images passed quality control checks for saturation and dynamic range and were cropped to the area of interest. The alignment procedure was semiautomated with approximately a dozen alignment vectors added manually per gel and then automatically aligned using the SameSpots alignment tool. Individual gel images were then grouped by sample type using the SameSpots between-subject experimental design. After the alignment was applied across all images, spots found to have ANOVA $P$ value $\leq 0.01$, Max fold change $\geq 2$, and unique upregulation in standardized expression profiles based on dendrogram grouping were selected for manual picking from the preparative gels for mass spectrometry analysis using the aligned DIGE scans as visual reference.

Two-dimensional picking gels were run for each of the three $D$. citri samples, and the spots identified as uniquely upregulated in one condition were excised from the three gels. For each target spot, a control spot was excised from a picking gel run with one of the other two samples. For the Murraya and Cmac gel spots, the Murraya host switch sample was run as the control picking gel; for the Murraya host switch gel spots, the Cmac sample was run as the control picking gel. Proteins were extracted from all target spot and control spot samples, and peptides from samples were prepared for mass spectrometry analysis.

2-D-DIGE mass spectrometry sample preparation. Excised gel plugs were washed in $50 \% 100 \mathrm{mM}$ ammonium bicarbonate and $50 \%$ acetonitrile $(\mathrm{ACN})$ and incubated for $10 \mathrm{~min}$ at room temperature before removing supernatant. Plugs were then washed in $\mathrm{ACN}$, incubated for $5 \mathrm{~min}$ at room temperature, and dried. Proteins were digested using $100 \mathrm{ng}$ of trypsin in $40 \mathrm{mM}$ of ammonium bicarbonate and $10 \% \mathrm{ACN}$ and placed on ice for $20 \mathrm{~min}$ to rehydrate before digesting overnight at $30^{\circ} \mathrm{C}$.

Supernatant was collected, and peptides were extracted from gel plugs by adding $50 \% \mathrm{ACN}$ and $2.5 \%$ formic acid and incubating for $45 \mathrm{~min}$ at room temperature. Samples were sonicated and briefly centrifuged, and extract was combined with earlier supernatant. A second extraction from gel plugs was performed using 90\% ACN and $0.1 \% \mathrm{FA}$ and incubated for $30 \mathrm{~min}$, and supernatant was again combined with earlier extracts. Extracted peptide samples were dried down before mass spectrometry analysis.

2-D-DIGE mass spectrometry data acquisition. An LTQ Orbitrap XL mass spectrometer (Thermo Fisher Scientific), equipped with a nano-ion spray source was coupled to an EASY-nLC system (Thermo Fisher Scientific). The nano-flow LC system was configured with a $180-\mu \mathrm{m}$ i.d. fused silica capillary trap column containing $3 \mathrm{~cm}$ of Aqua 5- $\mu \mathrm{m} \mathrm{C18}$ material (Phenomenex) and a self-pack PicoFrit $100-\mu \mathrm{m}$ analytical column with an $8-\mu \mathrm{m}$ emitter (New Objective, Woburn, MA) packed to $15 \mathrm{~cm}$ with Aqua 3- $\mu \mathrm{m}$ C18 material (Phenomenex). Mobile phase A consisted of $2 \%$ ACN/ $0.1 \%$ FA, and mobile phase B consisted of $90 \%$ ACN/0.1\% FA. Five microliters of each sample, dissolved in mobile phase A, was injected through the autosampler onto the trap column. Peptides were then separated using the following linear gradient steps at a flow rate of $400 \mathrm{~nL} / \mathrm{min}$ : $5 \% \mathrm{~B}$ for $1 \mathrm{~min}, 5 \% \mathrm{~B}$ to $35 \% \mathrm{~B}$ over $20 \mathrm{~min}, 35 \% \mathrm{~B}$ to $75 \% \mathrm{~B}$ over $5 \mathrm{~min}$, held at $75 \% \mathrm{~B}$ for $5 \mathrm{~min}$, $75 \% \mathrm{~B}$ to $8 \% \mathrm{~B}$ over $1 \mathrm{~min}$, and the final $5 \mathrm{~min}$ held at $8 \% \mathrm{~B}$.

Eluted peptides were electrosprayed into the LTQ Orbitrap XL mass spectrometer with the application of a distal $2.5-\mathrm{kV}$ spray voltage and a capillary temperature of $250^{\circ} \mathrm{C}$. Each full-scan mass spectrum $(\operatorname{Res}=60,000 ; 400$ to $1,700 \mathrm{~m} / \mathrm{z}$ ) was followed by MS/MS spectra for the top six masses. Collision-induced dissociation was used with the normalized collision energy set to 35 for fragmentation, the isolation width set to 3.0, and an activation time of $30 \mathrm{~ms}$. A duration of $90 \mathrm{~s}$ was set for the dynamic exclusion with an exclusion list size of 500, repeat count of 1 , and exclusion mass width of $10 \mathrm{ppm}$. We used monoisotopic precursor selection for charge states $\geq 2+$, and all data were acquired in profile mode.

MS/MS search parameters and acceptance criteria. Thermo *.raw files were converted into .mzML files using MSConvert in Proteowizard. Mascot Daemon 2.3.2 (Matrix Science, Boston, MA) was used to search .mzML files against a database comprising predicted proteins of D. citri (from Diaci psyllid genome assembly version 1.1) and its three known endosymbionts, Wolbachia, ' $C$. Profftella armatura', and ' $C$. Carsonella rudii'. The database also included common laboratory contaminant proteins (Hodge et al. 2013). MS/MS search parameters included ESI-TRAP instrument, fixed modifications (cysteine: carbamidomethyl), variable modifications (asparagine, glutamine: deamidated; methionine: oxidation), maximum of one missed cleavage, MS/MS tolerance of $\pm 0.5 \mathrm{Da}$, peptide tolerance of $\pm 30 \mathrm{ppm}$, and peptide charges of $2+$ and $3+$.

Protein quantitation in samples collected from protein gel spots. In the analysis of 2-D gels, more than one protein is typically identified per spot. To identify which proteins are changing in abundance within each 2-D gel spot, we used the 2-D GeLC approach (Cilia et al. 2011a; Yang et al. 2007). The exponentially modified protein abundance index (emPAI) value (Ishihama et al. 2005 ) is given for each protein in the Mascot search results. The mol fraction emPAI value was calculated for each protein as follows: mol fraction $=$ emPAI $/ \Sigma(\mathrm{emPAI})$ (Ishihama et al. 2005). The protein fraction represented by each protein in each spot was estimated by the mol fraction emPAI values. To compare the abundance of a given protein between the target sample in which the larger gel spot was initially identified and the control sample, the fractional spot volume was calculated, wherein the mol fraction emPAI value is multiplied by the DIGE spot volume calculated by fluorescent imaging of the initial Cy3 and Cy5 labeled gels (Cilia et al. 2011b). The $\log _{2}$ ratio of the fractional spot volume between each protein in the target spot and the control spot was calculated, and a $\log _{2}$ ratio of 1 was used as a threshold to select which proteins are more abundant in the target spots.

Label-free protein extraction and mass spectrometry sample preparation. Psyllid samples from the same three host plant sample classes analyzed by DIGE (Cmac, Murraya, Murraya host 
switch) as well as insect samples from the Cmac host switch class were analyzed in parallel using label-free shotgun proteomics. Three biological replicate insect samples (200 adult insects each) from each of the four sample classes were ground, and protein was precipitated in the same manner as the 2-D-DIGE samples. Protein pellets were solubilized in $8 \mathrm{M}$ of urea and $50 \mathrm{mM}$ of triethylammonium bicarbonate overnight at room temperature with agitation. Supernatant was collected after centrifugation, and protein concentration was determined using the Quick Start Bradford Protein Assay (Bio-Rad).

Protein samples were reduced by adding tris-carboxy-ethyl phosphine and incubating at $55^{\circ} \mathrm{C}$ for $1 \mathrm{~h}$. Samples were briefly centrifuged and allowed to cool to room temperature before cysteine alkylation using iodoacetamide. Cysteine alkylation was performed at room temperature in the dark for $1 \mathrm{~h}$. Samples were diluted to less than $1 \mathrm{M}$ of urea with triethylammonium bicarbonate before digestion with sequencing grade modified trypsin (Promega, Madison, WI) overnight at $37^{\circ} \mathrm{C}$.

Digested samples were dried and resuspended in $500 \mu \mathrm{l}$ of $0.1 \%$ FA in water before column clean-up. Oasis MCX columns were used with a Phenomenex vacuum manifold, with pressure kept between 4 and 5 in $\mathrm{Hg}$. Columns were conditioned with $1 \mathrm{ml}$ of methanol, $1 \mathrm{ml}$ of $3 \%$ ammonium hydroxide in water, and $2 \mathrm{ml}$ of methanol followed by $3 \mathrm{ml} 0.1 \%$ FA in water. Samples were loaded onto columns, and columns were then washed with $1 \mathrm{ml}$ of $0.1 \% \mathrm{FA}$ in water followed by $1 \mathrm{ml}$ of $0.1 \% \mathrm{FA}$ in methanol. Samples were eluted into a collection tube using $1 \mathrm{ml}$ of $3 \%$ ammonium hydroxide in methanol. Cleaned samples were dried down before mass spectrometry analysis.

Label-free mass spectrometry data acquisition and analysis. The dried tryptic digests were solubilized in $500 \mu \mathrm{l}$ of $0.1 \%$ trifluoracetic acid and $2 \% \mathrm{ACN}$ by vortexing for $10 \mathrm{~min}$ at $37^{\circ} \mathrm{C}$ and bath sonication for $5 \mathrm{~min}$ to give an estimated final concentration of $0.4 \mu \mathrm{g} / \mu \mathrm{l}$. The solubilized digests were centrifuged at $10,000 \times g$ for 5 min to pellet any particulates that might cause HPLC clogging, and then $50 \mu \mathrm{l}$ of each supernatant was placed into autosampler vials. The sample run order was randomized, with no blank runs in between.

All mass spectrometry was performed on an LTQ-Orbitrap-Fusion (Thermo Fisher Scientific). Three microliters $(\sim 1.2 \mu \mathrm{g})$ was loaded from the autosampler onto a $150-\mu \mathrm{m}$ Kasil fritted trap packed with ReproSil-Pur 120 A C18-AQ 3 micron beads (Dr. Maisch) to a bed length of $2 \mathrm{~cm}$ at a flow rate of $2 \mu \mathrm{l} / \mathrm{min}$ for $5 \mathrm{~min}$. After loading and desalting, the trap was brought in-line with a pulled fused-silica capillary tip (75- $\mu \mathrm{m}$ i.d.) packed with $30 \mathrm{~cm}$ of the same chromatography material mounted in a nanospray source with the column and trap heated to $50^{\circ} \mathrm{C}$. Peptides were eluted off the trap and column using a Waters Nanoacquity binary ultra performance liquid chromatography pump using a gradient of 5 to $13 \% \mathrm{ACN}$ in $0.1 \%$ FA over $10 \mathrm{~min}$, followed by 13 to $28 \%$ gradient over $40 \mathrm{~min}$, and then followed by 28 to $60 \%$ over $10 \mathrm{~min}$. The trap and column were subsequently washed for $5 \mathrm{~min}$ each with $60 \%$ and $95 \% \mathrm{ACN}$ in $0.1 \%$ $\mathrm{FA}$, all at a flow rate of $250 \mathrm{nl} / \mathrm{min}$.

The mass spectrometer was operated using electrospray ionization $(2 \mathrm{kV})$ with the heated transfer tube at $275^{\circ} \mathrm{C}$ using data-dependent acquisition in "Top Speed" mode, whereby one orbitrap mass spectrum $(\mathrm{m} / \mathrm{z}, 400$ to 1,600 with quadrupole isolation) was acquired with multiple linear ion trap tandem mass spectra every $3 \mathrm{~s}$ or less. The resolution for MS in the orbitrap was 120,000 at $\mathrm{m} / \mathrm{z}, 200$, and for MS/MS the linear ion trap provided unit resolution. The automatic gain control target for MS in the orbitrap was $2 \mathrm{e}^{5}$, whereas for MS/MS it was $1 \mathrm{e}^{4}$, and the maximum fill times were 50 and $35 \mathrm{msec}$, respectively. The MS/MS spectra were acquired using quadrupole isolation with an isolation width of $1.2 \mathrm{~m} / \mathrm{z}$ and higher energy collisional dissociation normalized collision energy of $30 \%$. The precursor ion threshold intensity was set to $5 \mathrm{e}^{3}$ to trigger an MS/ MS acquisition. Furthermore, MS/MS acquisitions were allowed for precursor charge states of 2 to 7 . Dynamic exclusion (including all isotope peaks) was set for $10 \mathrm{~s}$ using monoisotopic precursor selection with a mass error of $10 \mathrm{ppm}$. The fragment ions were analyzed in the linear trap using the "rapid" scan rate. Each sample was analyzed in duplicate, in which the orbitrap was used for MS/MS acquisition the second time. The MS/MS conditions for the orbitrap were similar, except that the resolution was set to 15,000, the target AGC was $5 \mathrm{e}^{4}$, and the maximum fill time was set to $22 \mathrm{msec}$.

Thermo *.raw files were converted into Mascot Generic Format (.mgf) using MSConvert in Proteowizard, and Mascot Daemon 2.3.2 was used to search .mgf files against the $D$. citri MCOT protein database (Saha et al. 2017). MS/MS search parameters for the samples run on the Orbitrap included ESI-FTICR instrument, fixed modifications (cysteine: carbamidomethyl), variable modifications (asparagine, glutamine: deamidated; methionine: oxidation), maximum of one missed cleavage, MS/MS tolerance of $\pm 0.02 \mathrm{Da}$, peptide tolerance of $\pm 25 \mathrm{ppm}$, and peptide charges of $2+, 3+$, and $4+$. MS/MS search parameters for the samples run on the Velos Linear trap included ESI-TRAP instrument, fixed modifications (cysteine: carbamidomethyl), variable modifications (asparagine, glutamine: deamidated; methionine: oxidation), maximum of one missed cleavage, MS/ MS tolerance of $\pm 0.6 \mathrm{Da}$, peptide tolerance of $\pm 25 \mathrm{ppm}$, and peptide charges of $2+, 3+$, and $4+$. Files with the *.dat extension were exported from Mascot and loaded into Scaffold Q+ (version 4.6.1, Proteome Software, Portland, OR) and used to calculate normalized spectral counts for each protein from each sample. Scaffold protein and peptide thresholds were set to $95 \%$, with a minimum of two peptides per protein. The Orbi and Linear datasets were merged together; once combined, they had a protein false discovery rate (FDR) of $0.8 \%$ and a peptide FDR of $0.07 \%$. Analysis of weighted spectral count data for identification of proteins differentially abundant among the four sample categories was performed in Scaffold using ANOVA (significance level $P<0.05$ using the Hochberg-Benjamini multiple test correction).

Mass spectrometry data availability. All mass spectrometry raw data have been deposited to the ProteomeXchange Consortium (Vizcaino et al. 2014) via the PRIDE partner repository (Vizcaino et al. 2016) with dataset identifier PXD011401.

\section{RESULTS}

D. citri host switch affects acquisition and transmission of CLas. Infection rates in D. citri adults were between 90 and $95 \%$, and average $\mathrm{Ct}$ values of 27 indicated high $C$ Las titer in $D$. citri colonies. Adult psyllids from $D$. citri colonies maintained on CLasinfected $C$. medica plants were transferred to healthy $M$. paniculata plants (host switch 1), where they were maintained for 6 and 12 months before transfer back to CLas-infected $C$. medica (host switches 2 and 3, respectively) (Fig. 1). Throughout the experiment, the percent infection (percentage of psyllids testing positive for $C$ Las by qPCR) and mean $\mathrm{Ct}$ value from $C$ Las qPCR of positive insects (for estimation of $C$ Las titer) were determined for insects continually maintained on CLas-infected $C$. medica plants. Two sets of founder insects from the same population (subcolonies A and B) were transferred from $C$ Las-infected $C$. medica plants to healthy $M$. paniculata plants. At three time points over the 4 months following host switch 1 , the percent infection and $\mathrm{Ct}$ values were determined for samples of $D$. citri collected from subcolonies A and B on M. paniculata. Although the percent infection of the colonies continually reared on CLas-infected $C$. medica remained $>93 \%$ throughout the experiment, with mean $\mathrm{Ct}$ values between 24 and 28 (Table 1), the percent infection of insects transferred to healthy $M$. paniculata decreased to $2.5 \%$ within 4 months, with mean $\mathrm{Ct}$ value of 37.82 (Table 2). The difference in both percent infection and $\mathrm{Ct}$ value between host switch $1 \mathrm{D}$. citri (of both subcolonies A and B) and positive control $D$. citri continuously reared on infected citron is highly significant as early as 38 days on $M$. paniculata $(P<0.001$; Tables 1 and 2$)$.

Following 6 and 12 months of rearing on $M$. paniculata, adult psyllids were transferred to CLas-infected $C$. medica plants, and the 
percent infection and CLas titer were determined in subsequent psyllid generations over 3 to 4 months. Approximately 1 month after host switch 2 (6 months on $M$. paniculata), the $D$. citri percent infection increased from $2.5 \%$ in $M$. paniculata colonies to $70.5 \%$ in colonies reestablished on $C$ Las-infected $C$. medica plants; after 3 months on infected plants, the average percent infection was $47.7 \%$ (Table 3; Fig. 2). These values are significantly less than the $>90 \%$ percent infection values from colonies continually reared on infected $C$. med$i c a$, as are the mean $\mathrm{Ct}$ values of the host switch psyllids $(P<0.0001$, Tables 1 and 3). Similarly, the percent infection and CLas titer in colonies initiated with psyllids transferred from $M$. paniculata back to infected plants after host switch 3 (12 months on $M$. paniculata) remained significantly below these values for psyllids continually reared on infected $C$. medica $(P<0.0001$; Table 4$)$. In a subsequent replicated experiment to confirm these findings, the $C$ Las titer of $D$. citri transferred back to infected $C$. medica from $M$. paniculata remained below the titer in psyllids continually reared on infected C. medica (Supplementary Table S1).

Two detached-leaf transmission assays were performed 49 and 112 days after host switch 3 (to infected citron after 12 months on $M$. paniculata), and the percentage of inoculated leaves infected with $C$ Las was 70 and $75 \%$, respectively, the average of which was significantly lower $(P<0.045)$ compared with $90 \%$ in leaves inoculated by the positive control psyllids continuously reared on infected citron (Table 5).

Host plant impact on diversity and abundance of psyllid proteoforms. We used DIGE, a top-down approach for the analysis of intact proteins, to identify protein spots from 2-D gels that were differentially expressed among $D$. citri reared on $M$. paniculata (sample class: Murraya), D. citri reared on C. macrophylla (sample class: Cmac), and D. citri transferred to $M$. paniculata for 5 days after long-term rearing on $C$. macrophylla (sample class: Murraya host switch). A representative gel scan showing protein spot pattern of the psyllid proteome is shown in Figure 3. The two gels depicted in Figure 3 represent a dye swap experiment in which duplicate Cmac and Murraya host switch protein samples are each labeled with $\mathrm{Cy} 3$ and $\mathrm{Cy} 5$ dyes and both combinations of labeled samples are run on replicate 2-D gels. A total of eight gels containing the three biological replicates from the three sample classes were run (Supplementary Fig. S1). Following fluorescence quantitation of all gel scans and statistical analysis, 114 spots were found to have ANOVA $P$ value $\leq 0.01$, Max fold change $\geq 2$, and unique upregulation in standardized expression profiles based on dendrogram grouping (Murraya: 45 spots; Cmac: 41 spots; Murraya host switch: 28 spots). These 114 spots were selected for manual picking from the preparative gels for mass spectrometry analysis (Supplementary Fig. S2).
Preparative gels were run for each sample, and target upregulated gel spots were excised from the appropriate positions in the gels. Control spots were excised from the same positions in gels on which control protein samples from one of the other two classes were run. Peptide samples prepared from excised gel spots were analyzed by mass spectrometry. No proteins were identified in six spots, but multiple proteins were identified in all other spots, resulting in a total of 866 unique proteins (Supplementary Table S2). The most abundant protein in each spot (based on mol fraction emPAI value, see Materials and Methods) is given in Supplementary Table S3.

The term proteoform is used to describe the structural and functional diversity of proteins produced from a single gene (Smith et al. 2013). In several cases, a single protein was identified as the most abundant protein in multiple distinct spots, a result that is consistent with proteoform expression. Three examples of this are hexamerin-like protein 4 (MCOT02134.0.CT), the most abundant protein in four spots enriched in Cmac samples, and in one spot enriched in Murraya host switch samples, cuticle protein 8 (MCOT02120.2.CC), the most abundant protein in three spots enriched in Cmac samples, and vitellogenin-1 (XP_008487106.1), the most abundant protein in five spots enriched in Murraya host switch samples (Fig. 4; Supplementary Table S3). Hexamerins are insect larval storage proteins related to the arthropod immunity proteins tyrosinase and hemocyanin (Burmester 2002, 2015; Decker and Jaenicke 2004), and vitellogenin is a lipid-binding protein with functions in reproduction and immunity documented in a range of insects (Burmester 2002; Salmela et al. 2015). The spots containing vitellogenin as the most abundant protein migrate similarly in one dimension (molecular weight) but resolve into distinct spots in the second dimension (isoelectric point). The three-dimensional spot volume representation for four vitellogenin spots in the three D. citri samples is shown in Figure 5. Vitellogenin and hexamerin play important roles in many aspects of insect biology, and the proteoform diversity of these proteins has been characterized in other insects, including the honeybee, in

TABLE 1. 'Candidatus Liberibacter asiaticus' (CLas) infection percentages and mean cycle threshold values for the positive control Diaphorina citri colony maintained on CLas-infected Citrus medica throughout the experiment

\begin{tabular}{lcc}
\hline CLas assay number: date & $\begin{array}{c}\text { \% CLas-infected } \\
\text { insects/total tested }\end{array}$ & Mean $\mathrm{Ct}^{\mathrm{a}}$ value \\
\hline 1: 24 Apr 2017 & $93.3(n=30)$ & 27.26 \\
2: 19 May 2017 & $96.7(n=30)$ & 28.0 \\
3: 21 Aug 2018 & $99.4(n=175)$ & 24.41 \\
\hline
\end{tabular}

${ }^{\mathrm{a}} \mathrm{Ct}$, cycle threshold.

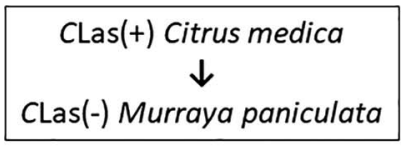

Host Switch \#1

$4 / 24 / 17$

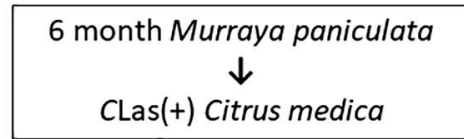

Host Switch \#2 (6 month)

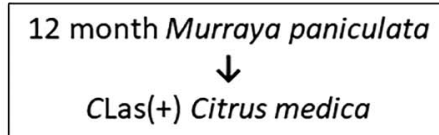

Host Switch \#3 (12 month) $10 / 24 / 17$

\section{$5 / 8 / 18$}

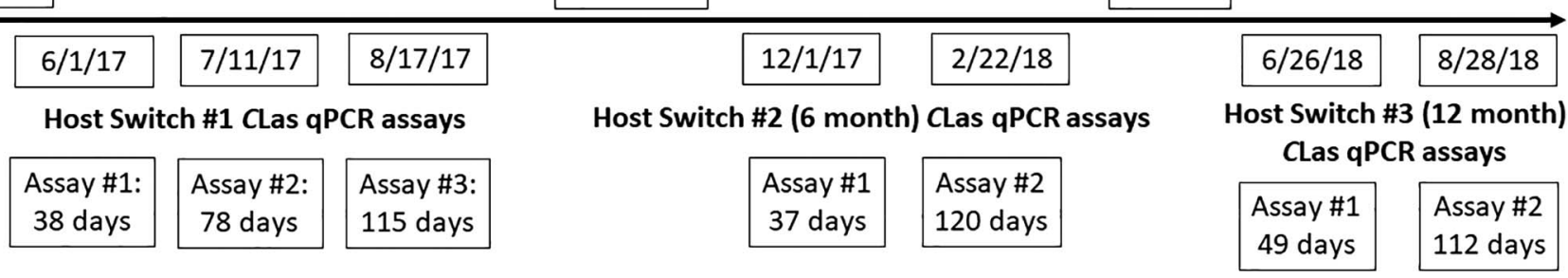

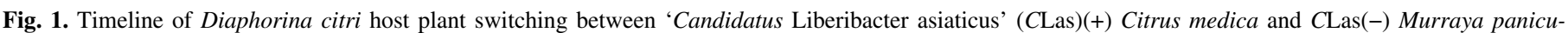

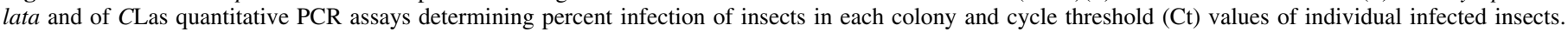

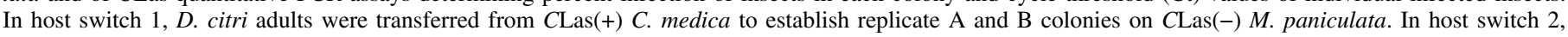

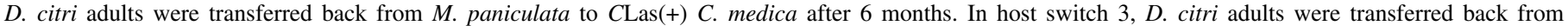
M. paniculata to CLas(+) C. medica after 12 months. 
which multiple proteoforms of vitellogenin and hexamerin were identified by DIGE (Erban et al. 2013).

The proteins identified as most abundant in each spot were grouped into functional categories based on GO terms and previous characterization of orthologous proteins in other organisms. A single functional representative functional category was assigned to the most abundant protein in each spot (Supplementary Table S3). Metabolism (13 proteins) and cytoskeleton (12 proteins) were the functional categories into which the most abundant protein identified in the Murraya-upregulated spots were grouped. Metabolism (12 proteins) and molecular chaperone (5 proteins) were the functional categories most commonly associated with the most abundant protein in the Murraya host switch-upregulated spots. Cuticle protein (12 proteins) and metabolism (6 proteins) were the most common functional categories associated with the most abundant protein identified in spots upregulated in Cmac samples (Supplementary Table S3).

Based on the calculation of fractional spot volumes in gel spot samples (see Methods), 469 unique proteins identified in all gel spots were found to be at least 2-fold more abundant in the target spot compared with the control spot. Many of these proteins were identified in multiple spots, likely representing different proteoforms of the same protein that migrate differently under 2-D electrophoresis. A total of 879 unique protein/spot combinations was identified as more abundant in one of the three samples (Supplementary Table S4).

Of these 469 proteins, 212 were found to be more abundant in the Murraya compared with the Murraya host switch sample; 108 proteins were found to be more abundant in the Cmac compared with the Murraya host switch sample, and 94 proteins were found to be more abundant in the Murraya host switch compared with the Cmac sample (Fig. 6, Supplementary Table S5). A number of proteins were found at higher levels in two of the samples than the third: 21 proteins were found at higher levels in both Murraya and Cmac compared with Murraya host switch spots, 17 proteins were found at higher levels in Murraya and Murraya host switch spots compared with $\mathrm{Cmac}$, and 10 proteins were found at higher levels in the Cmac and Murraya host switch spots compared with Murraya (Fig. 6; Supplementary Table S5). There were seven proteins for which specific proteoforms identified in different spots were found to be more abundant in each of the three sample classes compared with the others. The seven proteins in this category include proteins that have previously been characterized by diverse proteoforms, including actin (Wagner et al. 2002), malate dehydrogenase (Aranda et al. 2006), heat shock protein 70 (Ueda and Boettcher 2009), and the molecular chaperone DnaK (Rupprecht et al. 2007) produced by the Profftella endosymbiont of D. citri (Table 6).

Increased Wolbachia protein expression in Murraya-reared D. citri. Liquid chromatography and mass spectrometry were used for bottom-up (shotgun) proteomics analysis to complement the top-down (DIGE) analysis of the effect of host plant on protein expression in D. citri. In addition to the three sample classes analyzed by DIGE, insect samples transferred from Murraya to Cmac for 5 days feeding prior to collection (sample class: Cmac host switch) were included in this analysis.

Peptides mapping to a total of 2,001 proteins were identified from analysis of all four sample classes, with a protein FDR of $0.8 \%$ and a peptide FDR of $0.1 \%$ (Supplementary Table S6). The number of differentially expressed proteins identified in each pairwise comparison between insect sample classes is given in Table 7. The largest number of differentially expressed proteins (162) was identified between the Murraya and Cmac samples. There are relatively few proteins (only 27) differentially expressed between $\mathrm{Cmac}$ and Cmac host switch insects, whereas there are $>100$ proteins differentially expressed between Murraya and Murraya host switch insects, suggesting that insects transferred onto Cmac adjust to their new host plant more readily than those transferred onto Murraya. All proteins identified as differentially abundant between samples using the Fisher's exact test are given in Supplementary Table S7.

Proteins produced by the bacterial endosymbiont Wolbachia showed a consistent pattern of differential expression among D. citri sample classes. Five Wolbachia proteins were found to be differentially expressed among sample classes, and in all cases these proteins were upregulated in the Murraya samples. These five proteins include three proteins of unknown function, a membrane protein, and the molecular chaperone GroeL (Table 8). The Wolbachia proteins were identified in all biological samples, with spectral counts in Murraya samples two- to fourfold higher than in CMac and/or host switch samples. No conserved domains were identified in the three unknown Wolbachia proteins found to be upregulated in Murraya-reared D. citri samples.

Following published protocols (Chu et al. 2016), qPCR analysis of Wolbachia and D. citri genomic targets was performed on DNA extracted from Murraya and Cmac samples (three replicate samples of 10 insects each from each class). The copy number of both genes (Wolbachia ftsZ and D. citri $\mathrm{Wg}$ ) was calculated by comparison between $\mathrm{Ct}$ values of insect DNA samples and a dilution series of synthetic plasmids containing the qPCR target. The gene copy number ratio between Wolbachia and $D$. citri was significantly greater $(246$ compared with 18 , unpaired T-test $P$ value $<0.001)$ in

TABLE 2. Host switch 1: Decline in the proportion of 'Candidatus Liberibacter asiaticus' (CLas)-infected adult Diaphorina citri (subcolonies A and B), previously reared on CLas-infected Citrus medica, following host switch to CLas-uninfected Murraya paniculata plants on 24 April 2017

\begin{tabular}{|c|c|c|c|c|c|c|c|}
\hline \multirow[b]{2}{*}{ CLas assay number: date } & \multirow[b]{2}{*}{ Days on Murraya } & \multicolumn{3}{|c|}{$\%$ CLas-infected psyllids/total tested } & \multicolumn{3}{|c|}{ Mean $\mathrm{Ct}^{\mathrm{a}}$ value } \\
\hline & & A & B & Average & A & B & Average \\
\hline 1: 1 Jun 2017 & 38 & $19.00(n=100)$ & $14.00(n=100)$ & $16.50(n=200)$ & 37.08 & 35.72 & 36.40 \\
\hline 2: $11 \mathrm{Jul} 2017$ & 78 & $10.00(n=150)$ & $0.35(n=286)$ & $3.67(n=436)$ & 36.31 & 35.17 & 36.24 \\
\hline
\end{tabular}

${ }^{a}$ Mean percent infection and cycle threshold $(\mathrm{Ct})$ value in all three assays are significantly different $(P<0.0001)$ compared with positive control $D$. citri continuously reared on CLas-infected citron (assays 1 to 3 in Table 1).

TABLE 3. 'Candidatus Liberibacter asiaticus' (CLas) quantitative PCR data on Diaphorina citri after host switch 2 (on healthy Murraya paniculata for 6 months then transferred to CLas-infected Citrus medica on 24 October 2017)

\begin{tabular}{|c|c|c|c|c|c|c|}
\hline \multirow[b]{2}{*}{ CLas assay number: date } & \multicolumn{3}{|c|}{$\%$ CLas-infected psyllids/total tested } & \multicolumn{3}{|c|}{ Mean $\mathrm{Ct}^{\mathrm{a}}$ value } \\
\hline & A & $\mathrm{B}$ & Average & A & B & Average \\
\hline 1: 1 Dec 2017 & $80.0(n=100)$ & $61.0(n=100)$ & $70.5(n=200)$ & 31.90 & 34.03 & 32.97 \\
\hline
\end{tabular}

${ }^{a}$ Mean percent infection $\left(X^{2}=31.24 ; P<0.0001\right)$ and cycle threshold $(\mathrm{Ct})$ value $(\mathrm{F}=66.71 ; P<0.0001)$ results are significantly different compared with positive control $D$. citri continuously reared on CLas-infected citron (assays 1 to 3 in Table 1). 


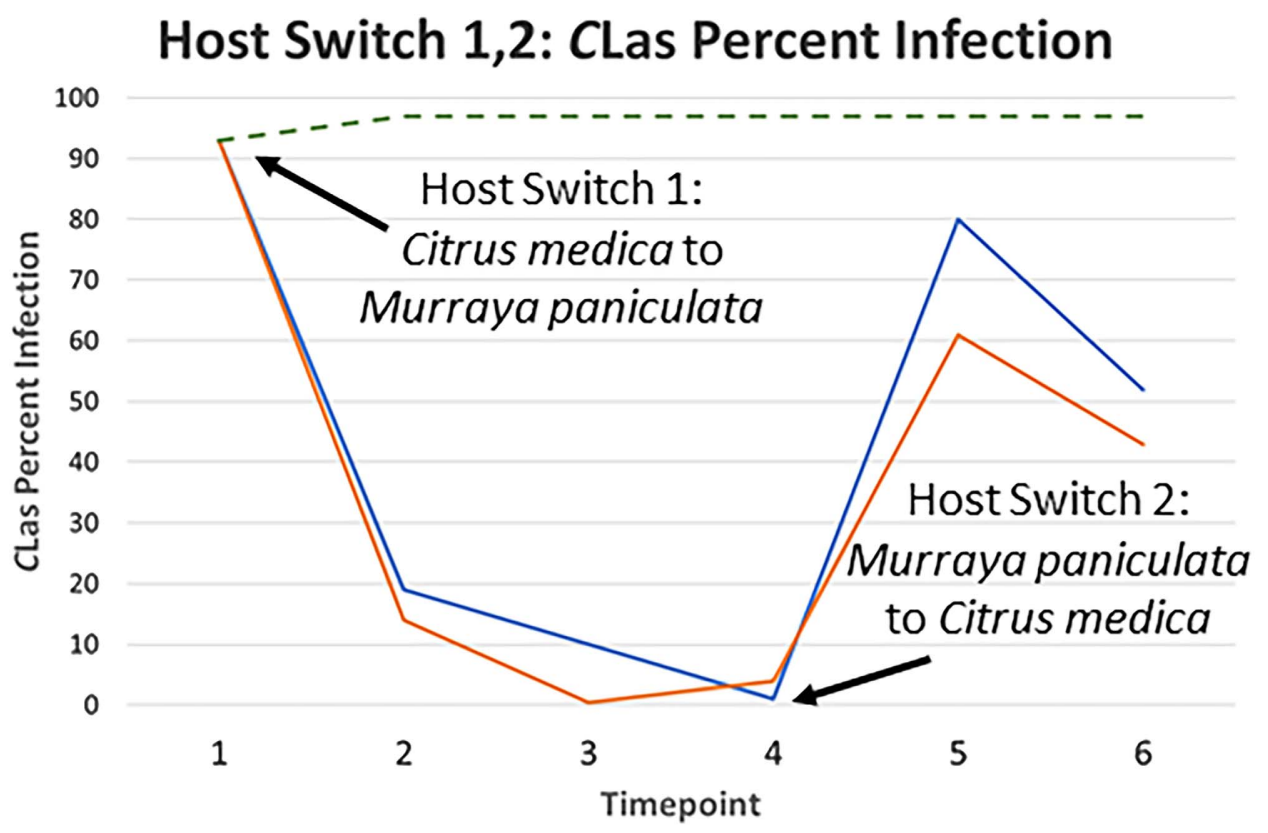

- Host Switch 6 month (A) - Host Switch 6 month (B) - -Clas(+) C. medica (control)

\section{Host Switch 1,2: CLas Ct Value}

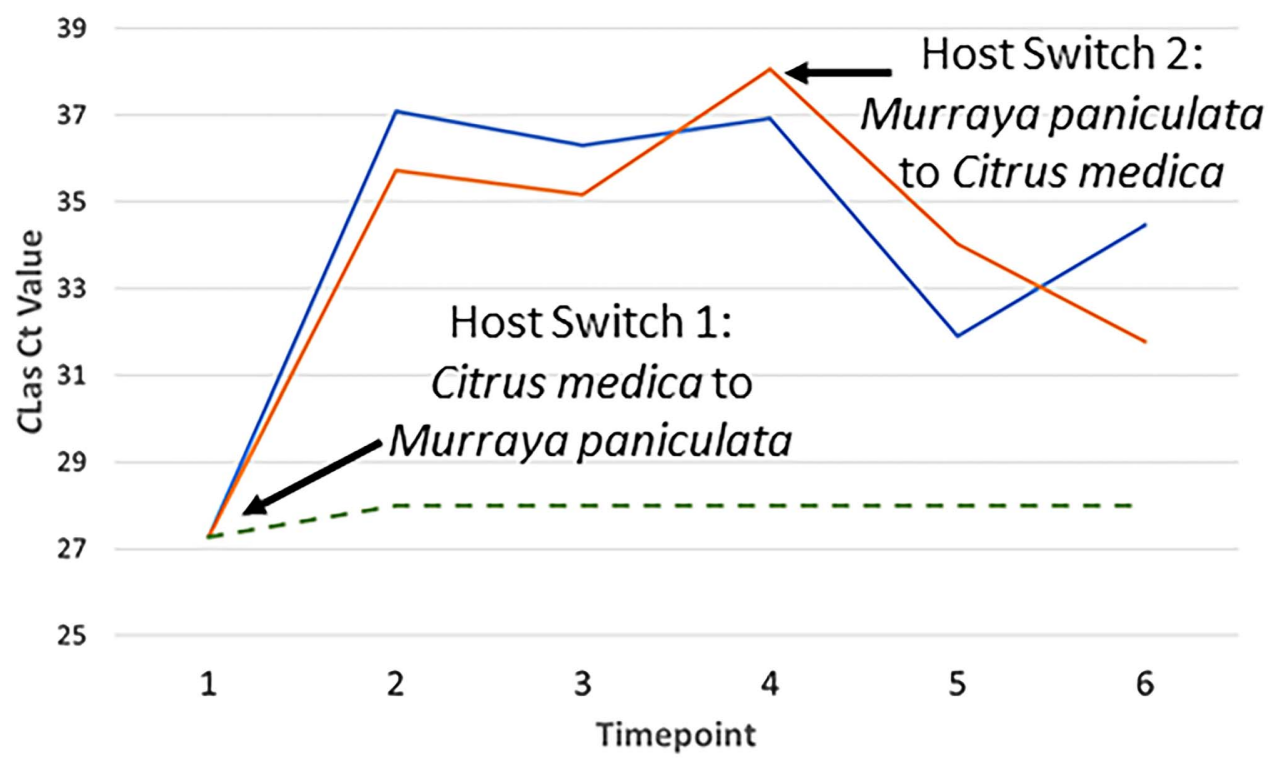

— Host Switch 6 month (A) — Host Switch 6 month (B) - -Clas(+) C. medica (control)

Fig. 2. Graphic representation of the impact of host switch 1 and 2 on the 'Candidatus Liberibacter asiaticus' (CLas) percent infection and cycle threshold (Ct) value in Diaphorina citri. Percent infection and Ct values of host switch colonies A and B represented by solid lines (colony A in blue and colony B in orange). Percent infection and $\mathrm{Ct}$ values of control D. citri continually reared on CLas(+) Citrus medica is represented by dashed line. Host switch 1 occurred at Timepoint 1, and host switch 2 occurred at Timepoint 4.

TABLE 4. 'Candidatus Liberibacter asiaticus' (CLas) quantitative PCR data on Diaphorina citri after host switch 3 (on healthy Murraya paniculata for 12 months then transferred to CLas-infected Citrus medica on 8 May 2018)

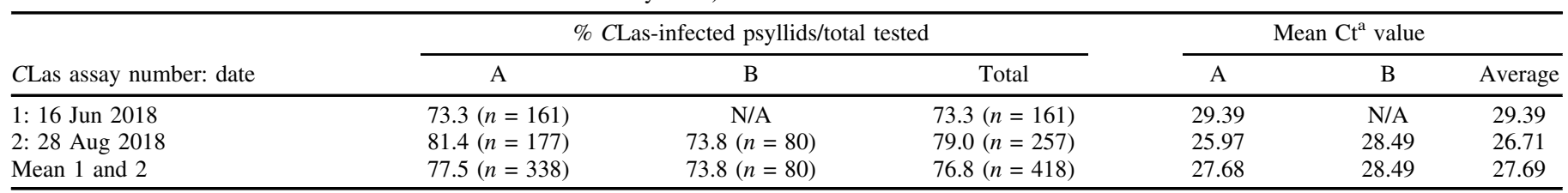

${ }^{a}$ Mean percent infection $\left(X^{2}=44.19 ; P<0.0001\right)$ and cycle threshold $(\mathrm{Ct})$ value $(\mathrm{F}=41.21 ; P<0.0001)$ results are significantly different compared with positive control D. citri continuously reared on CLas-infected citron (assay 3 in Table 1). 
Murraya compared with Cmac samples (Supplementary Table S9). This increase in the titer of Wolbachia in D. citri reared on Murraya compared with $\mathrm{Cmac}$ is consistent with the observation that Wolbachia proteins are more abundant in Murraya-reared insects. However, in a subsequent comparison of Wolbachia titer between $D$. citri colonies reared on Murraya and $C$. medica, the gene copy number ratio between Wolbachia and $D$. citri was greatly reduced $(<1)$ and was not significantly different between host plant sample classes (Supplementary Table S9). The relationship between Wolbachia titer and $D$. citri host plant is therefore complex and warrants further investigation.

Integration of top-down and bottom-up proteomics. We identified the proteins whose expression in $D$. citri is found by both topdown and bottom-up proteomics experiments to differ depending on the insect's host plant. These proteins represent the overlap between the differentially expressed proteins identified from mass spectrometry analysis of DIGE spots (based on $\log _{2}$ ratio $\geq 1$ of fractional spot volume between target and control spot; Supplementary Table S5) and from shotgun peptide mass spectrometry (based on Fisher's exact test on spectral count values; Supplementary Table S7).

For the Murraya-reared insects, 13 proteins were identified as upregulated by both DIGE and shotgun proteomics analysis (Table 9). Five of these 13 proteins are predicted to function in the cytoskeleton, two proteins play a role in cellular energy metabolism (ATP synthase alpha and beta subunits), and three others have unknown function. The final three proteins independently identified by both proteomics workflows as upregulated in Murraya-reared $D$. citri are an aldehyde dehydrogenase, a membrane protein from the Wolbachia endosymbiont, and a protein annotated as $\mathrm{N}$-acetylmuramoyl-L-alanine amidase, which is predicted to catabolize peptidoglycan in bacterial cell walls (Mellroth and Steiner 2006) (Table 9).

For the Cmac-reared insects, nine proteins were identified as upregulated by both DIGE and shotgun proteomics analysis (Table 10). Four of these proteins are predicted to be metabolic storage proteins known as hexamerins (including hemocyanin 2), and two others are cuticle proteins (including skin secretory protein xP2). The other proteins upregulated in Cmac-reared insects include a protein involved in Ras GTPase signaling, an RNA polymerase, and a protein of unknown function.

For the Murraya host switch insects, the five proteins identified as upregulated by both DIGE and shotgun proteomics analysis include four proteins with immune-related function and a protein of unknown function (Table 11). The immune-related proteins include two transferrins, which bind and transport iron, a vitellogenin, and hemocyanin 1 , which has been shown to physically interact with a $C$ Las protein and be upregulated in $D$. citri exposed to CLas (Ramsey et al. 2017).

\section{DISCUSSION}

In the generations following host switching of insects from CLasinfected $C$. medica plants to healthy $M$. paniculata, the percent infection of a colony of $D$. citri steadily decreased from $>90 \%$ to $2.5 \%$ (Tables 1 and 2). It is known that although Murraya plants are more resistant to $C$ Las compared with citrus, $C$ Las can multiply somewhat in Murraya, and D. citri can acquire it to a lower degree from Murraya compared with citrus plants (Walter et al. 2012a, b). However, it is still remarkable that 1 to $4 \%$ of $D$. citri adults tested were still CLas-infected after 4 months of rearing on healthy Murraya plants,

TABLE 5. Detached leaf transmission assay quantitative PCR data after host switch 3 (Diaphorina citri on healthy Murraya paniculata for 12 months and then transferred to 'Candidatus Liberibacter asiaticus' (CLas)-infected Citrus medica on 8 May 2018)

\begin{tabular}{|c|c|c|c|}
\hline D. citri sample group & $\begin{array}{l}\text { Transmission } \\
\text { assay date }\end{array}$ & $\begin{array}{c}\text { \% CLas-infected leaves/ } \\
\text { total tested }\end{array}$ & $\begin{array}{l}\text { Mean } \mathrm{Ct}^{\mathrm{a}, \mathrm{b}} \text { value } \\
\text { (infected leaves) }\end{array}$ \\
\hline Host switch 3 & 19 Jun 2018 & $70(n=20)$ & 35.44 \\
\hline Host switch 3 & 21 Aug 2018 & $75(n=20)$ & 33.95 \\
\hline Control (CLas+ C. medica) & 21 Aug 2018 & $90(n=20)$ & 34.04 \\
\hline
\end{tabular}

${ }^{a}$ Mean percent infected leaves $\left(X^{2}=4.02 ; P=0.045\right)$ results are significantly different between host switch 3 and control samples.

${ }^{\mathrm{b}} \mathrm{Ct}$, cycle threshold.

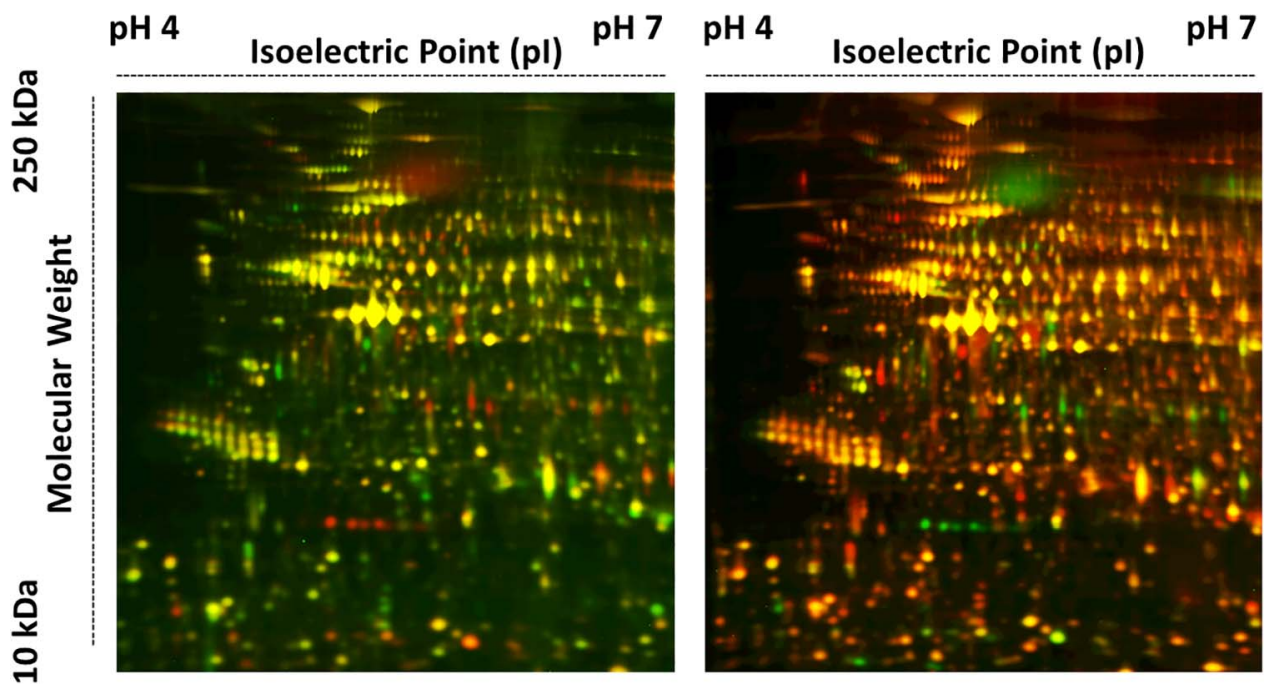

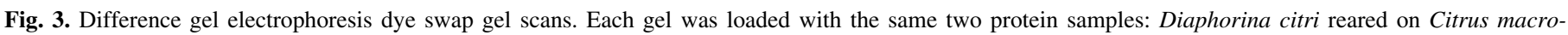

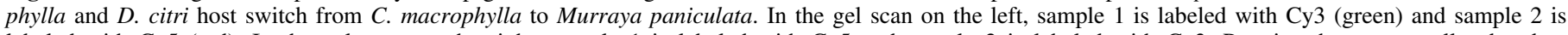

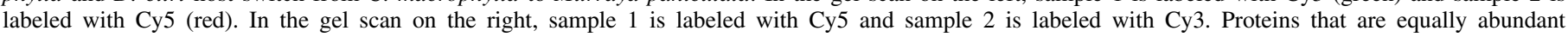

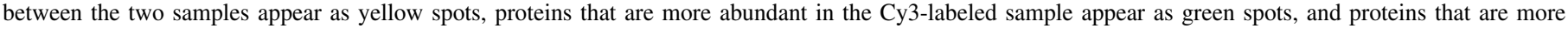
abundant in the Cy5-labeled sample appear as red spots. 


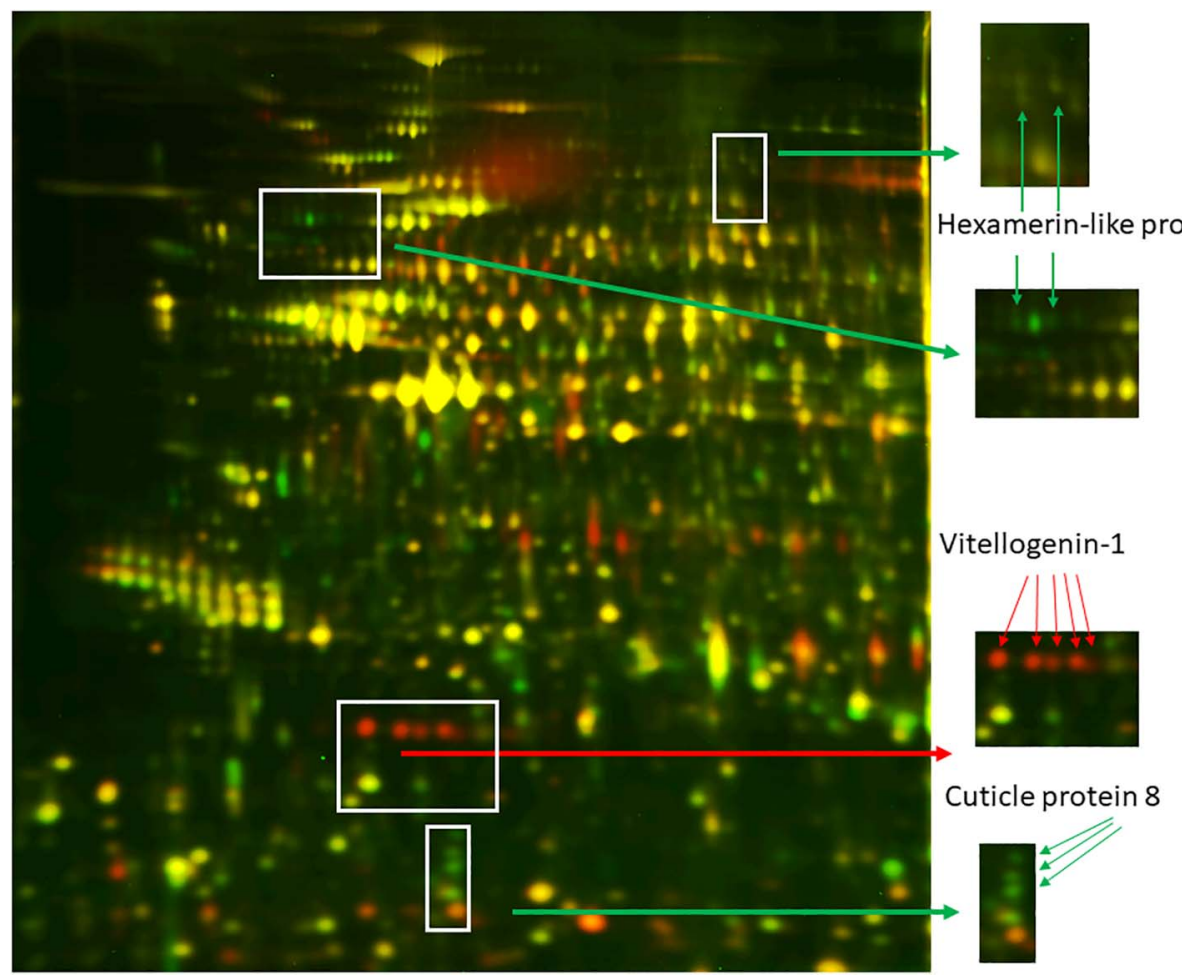

Fig. 4. Difference gel electrophoresis gel scan showing proteoform diversity. Multiple proteoforms of hexamerin-like protein 4, vitellogenin-1, and cuticle protein 8 were found to be more abundant in Diaphorina citri reared on Citrus macrophylla (green spots) or Murraya paniculata (red spots).
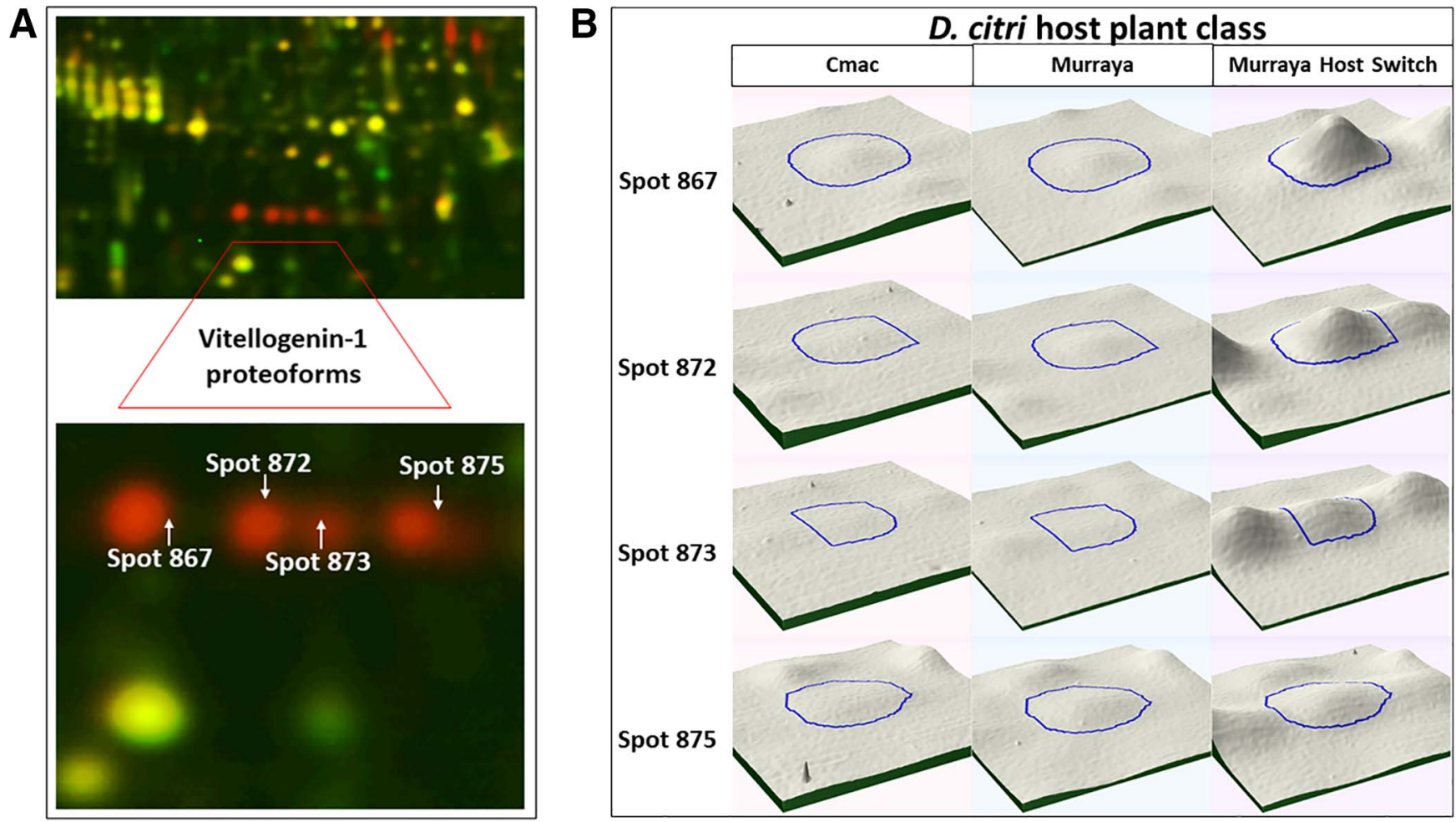

Fig. 5. Difference gel electrophoresis fluorescent gel scan and three-dimensional spot volume representation of four unique vitellogenin-1 proteoforms in Diaphorina citri protein samples. A, Fluorescent gel scan with protein samples from D. citri reared continuously on Citrus macrophylla (Cmac) labeled in green and protein samples from D. citri reared on Murraya paniculata for 10 days following host switch from C. macrophylla (Murraya host switch) labeled in red. The most abundant protein in spots $867,872,873$, and 875 are vitellogenin-1 proteoforms, and the red spot color indicates that the proteins in these spots are more abundant in Murraya host switch samples. B, Three-dimensional spot volume representation of spots 867, 872, 873, 875 in Cmac, Murraya (D. citri reared continuously on M. paniculata), and Murraya host switch samples. 
which could be significant epidemiologically if these infected psyllids move from Murraya to the more CLas-susceptible citrus plants in citrus-growing regions. This residual infectivity may be caused by either transovarial transmission of CLas to a small proportion in the progeny of infected psyllids as reported earlier (Walter et al. 2012a) or D. citri nymphs acquiring CLas from localized infected areas of Murraya plants where their infected mothers have been feeding, as reported earlier with citrus plants (Lee et al. 2015). Interestingly, our study demonstrates that the impact of Murraya on CLas acquisition and transmission by $D$. citri endures for several generations after transfer of insects back to $C$ Las-infected citrus. The lasting impact of this host plant effect on $C$ Las acquisition and transmission provides support for disease management strategies employing Murraya plants as part of a trap cropping system proximal to cultivated citrus at risk of $C$ Las infection by endemic $D$. citri populations.

Our results also demonstrate that the host plant of $D$. citri has a substantial impact on the insect proteome profile. Variable proteoform expressions, in which factors such as splicing and posttranslational modifications generate diversity in proteins produced from a single genetic locus (Smith and Kelleher 2018), were observed between insects from the different host plant sample classes. The importance of proteoform expression in pathogen transmission by insect vectors has previously been demonstrated in populations of the aphid Schizaphis graminum varying in their ability to transmit yellow dwarf viruses (Cilia et al. 2011b). The application of a top-down proteomics workflow alongside bottom-up proteomics provided the opportunity to visualize the diversity of proteoforms associated with a single protein within and between insect samples. For example, bottom-up (shotgun) proteomics analysis used spectral counting to reveal that peptides derived from the actin protein MCOT00135.1.CC were present in significantly greater abundance

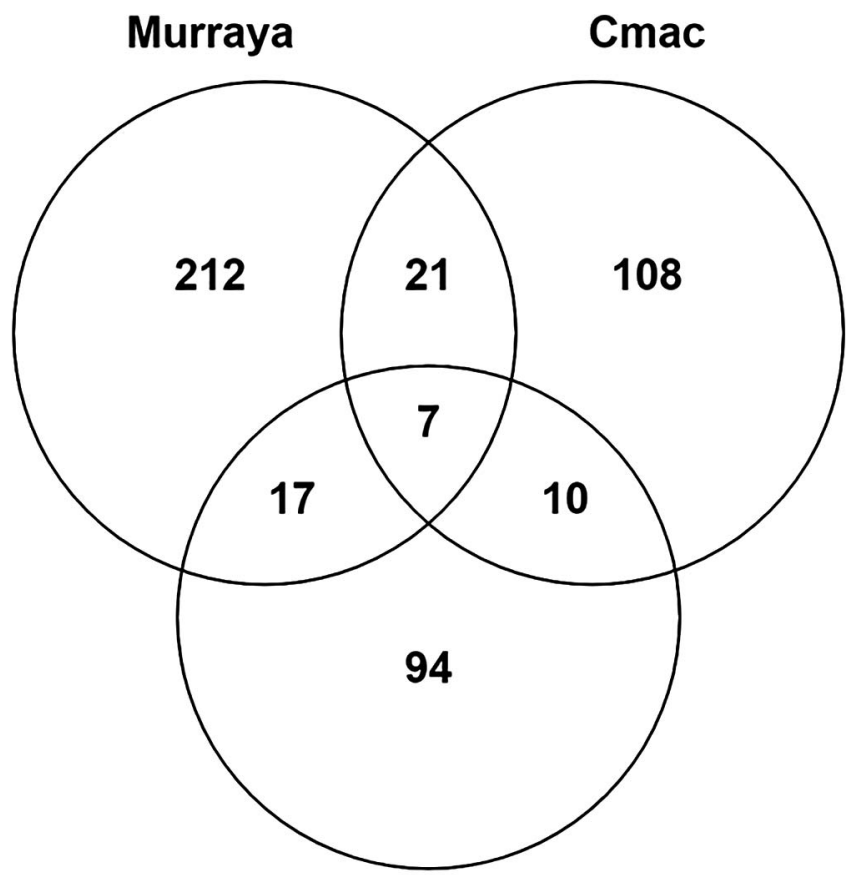

\section{Murraya Host Switch}

Fig. 6. Venn diagram representation of the number of proteins identified as upregulated in each insect sample class by mass spectrometry analysis of selected DIGE spots. The regions of the diagram representing the overlap of two or all three sample classes include those proteins identified as upregulated in spots from multiple sample classes. For these proteins, different isoforms were identified in multiple DIGE spots (Table 6). Insect sample classes: Murraya (Diaphorina citri reared continuously on Murraya paniculata), Cmac (D. citri reared continuously on Citrus macrophylla), Murraya host switch (D. citri reared on M. paniculata for 10 days after host switch from C. macrophylla). in Murraya-reared D. citri compared with the other sample classes. However, top-down (DIGE) proteomics analysis indicated that this actin protein exists as dozens of proteoforms, some of which are found at higher levels in Cmac and Murraya host switch samples compared with Murraya. Following statistical analysis of gel spot intensity in replicate 2-D gels with Cy3- and Cy5-labeled D. citri protein samples, a total of 114 gel spots was selected as having significantly greater DIGE spot volume in one sample compared with the other two. Peptides derived from the actin protein MCOT00135.1.CC were identified in 73 of these spots (Supplementary Table S2), and this protein was found to be the most abundant protein in eight of these spots (Supplementary Table S3). The actin proteins identified in these 73 spots are considered different proteoforms based on the fact that they migrate differently under 2-D electrophoresis. Unique actin proteoforms were determined to be present at significantly higher levels in the target compared with control sample for 43 of the 73 spots in which they were identified. Unique actin proteoforms were upregulated in all three sample classes: Cmac (12 spots), Murraya (19 spots), and Murraya host switch (12 spots). Whereas bottom-up proteomics methods have been used to characterize proteoforms with varying patterns of posttranslational modifications (Zheng et al. 2012), proteoform diversity is often homogenized in shotgun proteomics analysis of complex samples because it can be difficult to identify peptides that are specific to quantify and identify each proteoform. These findings demonstrate that details on changes in expression of specific proteoforms can be captured through top-down platforms such as DIGE.

Proteome complexity leads to the identification of more than one protein per spot. The most abundant protein in each spot is likely the differentially expressed protein that led to the original selection of the gel spot as variable in size between the sample classes. Mascot emPAI values were used to calculate the percentage of each spot comprising each protein, which was identified by mass spectrometry (Cilia et al. 2011b). For 24 of the 114 DIGE spots, the most abundant protein comprised $>50 \%$ of the total spot protein (Supplementary Table S3). These include five Murraya-upregulated spots for which the most abundant protein is a tropomyosin or troponin proteoform. A total of 12 spots selected by DIGE as upregulated in Murraya samples had a cytoskeleton protein as the most abundant spot (Supplementary Table S3): five were proteoforms of the MCOT00135.1.CC actin protein, five were troponin or tropomyosin proteoforms, one was a tubulin, and one was the PDZ and LIM domain protein ZASP (MCOT16492.1.CO), which has a documented function in cell adhesion and muscle structure. Additional evidence that cytoskeleton proteins play a significant role in the adaptation of $D$. citri to feeding on Murraya comes from the shotgun proteomics data, in which 25 Murraya-upregulated proteins have annotated function in the cytoskeleton and muscle structure (Supplementary Table S8). Five of these cytoskeleton proteins were found to be upregulated in Murraya samples by both DIGE and shotgun proteomics (Table 9).

Of the two host switch conditions, DIGE analysis was only performed on the Murraya host switch samples, and the five proteins found by both DIGE and shotgun proteomics to be upregulated in these samples include four that function in metabolism and immunity and one with unknown function (Table 11). One of these proteins (XP_008487106.1) is annotated as a vitellogenin, a large family of lipid-binding proteins with functions in immunity and reproduction. Several $D$. citri vitellogenins have been identified as among the most abundant proteins in the hemolymph (blood) of adult insects, including three that were found at higher levels in insect colonies collected from CLas-infected plants (Kruse et al. 2018). The other immunerelated proteins found by both DIGE and shotgun proteomics to be more abundant in Murraya host switch samples, hemocyanin 1 and two related transferrins, were also previously found to be upregulated in CLas-exposed insects (Ramsey et al. 2017).

In the bottom-up shotgun proteomics analysis, more changes in protein abundance were observed between Murraya-reared and host switch insects than between Cmac-reared and host switch insects. Whereas 27 proteins were found to be differentially abundant 
between insects continuously reared on Cmac and those collected from Cmac 5 days after being transferred from Murraya, 102 proteins were identified at significantly different levels between insects continuously reared on Murraya and those collected from Murraya 5 days after being transferred from Cmac (Table 7). These data support the hypothesis that $D$. citri transferred to Cmac from Murraya quickly adjust their physiology and metabolism such that their proteome profile is largely similar to insects continuously reared on Cmac. In contrast, the proteome of $D$. citri 5 days after transfer to Murraya from $\mathrm{Cmac}$ is more similar to Cmac-reared than Murraya-reared insects, which suggests a slower change in proteome regulation to the new host plant. Insect adaptation to host plant factors specific to Murraya may lead to substantial changes that occur over a longer time frame than the 5-day host switch period, whereas $D$. citri appears to rapidly adapt to feeding on $\mathrm{Cmac}$ after transfer from Murraya.

TABLE 6. Proteins for which unique isoforms were found to be upregulated in each of the three insect sample classes analyzed by difference gel electrophoresis ${ }^{a}$

Protein ID

WP_020915489.1

Molecular chaperone DnaK

(Candidatus Profftella armatura)

MCOT00093.0.CC

MCOT10574.0.MT

MCOT00135.1.CC

MCOT11909.0.MM

XP 008471373.1

MCOT18002.0.CT

Heat Shock Protein 70

Malate dehydrogenase

Actin

Actin

Actin-1

Endocuticle structural glycoprotein SgAbd-9

${ }^{a}$ Cmac, Diaphorina citri continually reared on Citrus macrophylla; sample class: Murraya, D. citri continually reared on Murraya paniculata; Murraya host switch, D. citri reared on $M$. paniculata for 10 days after host switch from C. macrophylla.

TABLE 7. Number of differentially abundant proteins identified in pairwise comparisons between all sample classes based on spectral counting of shotgun proteomics data (Fisher's exact test Benjamini-Hochberg adjusted $P$ value $<0.05$

\begin{tabular}{lc}
\hline Insect host comparison $^{\text {a }}$ & $\begin{array}{c}\text { Number of differentially } \\
\text { abundant proteins }\end{array}$ \\
\hline Murraya versus Murraya host switch & 102 \\
Murraya versus Cmac host switch & 107 \\
Murraya versus Cmac & 162 \\
Cmac versus Cmac host switch & 27 \\
Cmac versus Murraya host switch & 29 \\
Cmac host switch versus Murraya host switch & 40
\end{tabular}

${ }^{a}$ Cmac, Diaphorina citri continually reared on Citrus macrophylla; sample class: Murraya, D. citri continually reared on Murraya paniculata; Cmac host switch, D. citri reared on C. macrophylla for 10 days after host switch from M. paniculata; Murraya host switch, D. citri reared on M. paniculata for 10 days after host switch from C. macrophylla.
The largest number of protein changes in the bottom-up proteomics analysis was observed between $D$. citri continuously reared on Cmac or Murraya, including upregulation in Murraya-reared insects of several interacting proteins with coordinated roles in invertebrate muscle function, including actin, the actin-binding protein filamin, the calcium-binding protein sarcalumenin, myosin, paramyosin, troponin, titin, and twitchin (Supplementary Table S8). These changes are likely to have an impact on basic aspects of insect physiology, with potential consequences on the vectoring ability of the insect. Variation in insect morphology has previously been reported between $D$. citri reared on Murraya and Cmac, with significantly narrower wing shape observed in Murraya-reared insects (Paris et al. 2016). It is likely that the differential expression of cytoskeleton and muscle proteins that we have described between insects reared on these host plants could contribute to these observed morphological changes. Other morphological variation within $D$. citri populations that have been associated with vectoring ability is abdominal color or adult insects, with blue insects having the capacity for longer duration flight compared with gray insects collected from the same population (Martini et al. 2014). Our lab previously discovered that blue $D$. citri have higher expression of the gene encoding hemocyanin 1, a protein that uses copper to bind oxygen (Ramsey et al. 2017). The ortholog of this protein confers blue color to the hemolymph of horseshoe crabs and other arthropods and functions in metabolism and immunity (Burmester 2002; Decker and Jaenicke 2004; Lee et al. 2003).

Insect cuticle proteins were identified by DIGE-MS as the most abundant protein in $12 \mathrm{Cmac}$-upregulated spots, including three distinct spots where the most abundant proteins were proteoforms of the same cuticle protein (cuticle protein 8, MCOT02120.2.CC; Supplementary Table S3). Two cuticle proteins, skin secretory protein XP2 and pupal cuticle protein $78 \mathrm{E}$, were identified as upregulated in Cmac samples by DIGE and shotgun proteomics (Table 10). In contrast, although three distinct proteoforms of cuticle protein 8 were identified as Cmac-upregulated, this protein was found by spectral counting of shotgun proteomics data to be present at comparable levels in all insect sample classes. These data suggest that differential production of cuticle protein proteoforms is a component of the adaptation of $D$. citri to different host plant environments. Consistent with this hypothesis, the $D$. citri endocuticle structural glycoprotein SgAbd-9 (MCOT18002.0.CT) is among the seven proteins for which unique proteoforms were found by DIGE to be upregulated in each sample class (Table 6). Similar to cuticle protein 8 , this endocuticle glycoprotein was not identified as differentially expressed by shotgun proteomics. Both cuticle proteins are annotated as members of the protein family Pfam00379, indicating that they contain the Chitin bind_4 domain, also known as the R\&R consensus (Rebers and Riddiford 1988). Proteins containing this domain are classified in the CPR family, the largest family of cuticle proteins in arthropods, and contain subgroups RR-1 and RR-2 (Willis 2010). The structural and functional diversity of these proteins is illustrated by the fact that

TABLE 8. Wolbachia proteins identified as differentially abundant between Diaphorina citri sample classes ${ }^{\mathrm{a}}$

\begin{tabular}{|c|c|c|c|c|c|c|c|}
\hline \multirow[b]{2}{*}{ Protein ID } & \multirow{2}{*}{$\begin{array}{l}\text { Higher abundance } \\
\text { sample }\end{array}$} & \multirow{2}{*}{$\begin{array}{l}\text { Lower abundance } \\
\text { sample }\end{array}$} & \multicolumn{4}{|c|}{ Average spectral count by class $(n=3)$} & \multirow[b]{2}{*}{ Protein description } \\
\hline & & & Murraya & Murraya HS & $\mathrm{Cmac}$ & $\overline{\mathrm{Cmac} H S}$ & \\
\hline WP_017531978.1 & Murraya, Murraya HS & Cmac & 22.66 & 23.66 & 9 & 16 & Unknown protein \\
\hline WP_017531759.1 & Murraya & Cmac, Cmac HS & 11.33 & 4.666 & 2.33 & 2 & Unknown protein \\
\hline WP_026092655.1 & Murraya & Cmac, Cmac HS & 21 & 15 & 11.33 & 10 & Membrane protein \\
\hline WP_017531621.1 & Murraya & Cmac & 14.02 & 6.27 & 5.05 & 9.235 & $\begin{array}{l}\text { Molecular chaperone } \\
\text { GroeL }\end{array}$ \\
\hline WP_017531866.1 & Murraya & Cmac HS & 6 & 4.666 & 3 & 1.33 & Unknown protein \\
\hline
\end{tabular}

${ }^{a}$ Cmac, D. citri continually reared on Citrus macrophylla; sample class: Murraya, D. citri continually reared on Murraya paniculata; Cmac host switch, D. citri reared on C. macrophylla for 10 days after host switch from $M$. paniculata; Murraya host switch, D. citri reared on $M$. paniculata for 10 days after host switch from $C$. macrophylla. All differentially abundant Wolbachia proteins are found at higher levels in Murraya and/or Murraya host switch (HS) compared with Cmac and/or Cmac HS samples. Average spectral count data for each protein are given for each sample class. For each differentially abundant protein, spectral count values for statistically different higher abundance and lower abundance samples are in bold (Fisher's exact test Benjamini-Hochberg adjusted $P$ value $<0.05$ ). 
TABLE 9. Proteins found by both shotgun proteomics and difference gel electrophoresis to be upregulated in Murraya paniculata-reared Diaphorina citri ${ }^{\text {a }}$

\begin{tabular}{|c|c|c|c|}
\hline Protein ID & Protein description & $\begin{array}{l}\text { Spectral count } \log _{2} \text { fold change (lower } \\
\text { abundance sample under comparison) }\end{array}$ & $\begin{array}{l}\text { Fractional spot volume } \log _{2} \text { fold change } \\
\text { (Murraya versus Murraya HS), spot ID }\end{array}$ \\
\hline MCOT15074.1.CT & Unknown protein & $\begin{array}{l}1.7 \text { (Cmac) } \\
1.23 \text { (Murraya HS) }\end{array}$ & 6.2 , spot 923 \\
\hline XP_008471512.1 & Paramyosin & $\begin{array}{l}0.16 \text { (Cmac) } \\
0.31 \text { (Murraya HS) }\end{array}$ & 4.1 , spot 489 \\
\hline XP_008475404.1 & Myosin heavy chain, muscle-like & $\begin{array}{l}0.19 \text { (Cmac) } \\
0.27 \text { (Murraya HS) } \\
0.27 \text { (Cmac HS) }\end{array}$ & 6.2 , spot 489 \\
\hline MCOT01082.0.CT & ATP synthase subunit alpha & $0.16(\mathrm{Cmac})$ & 2.2 , spot 702 \\
\hline MCOT02123.0.CT & ATP synthase subunit beta & 0.09 (Cmac) & 4.8 , spot 617 \\
\hline MCOT03961.0.CT & Alpha-actinin, sarcomeric & 0.32 (Cmac) & 5.4 , spot 671 \\
\hline MCOT04879.1.CO & Unknown protein & $3.32(\mathrm{Cmac})$ & 2.3 , spot 624 \\
\hline MCOT15977.2.CC & Unknown protein & $\begin{array}{l}1.85 \text { (Cmac) } \\
1.25 \text { (Cmac HS) }\end{array}$ & 7.1 , spot 568 \\
\hline MCOT21051.0.CC & Tubulin beta- 1 chain & $0.24(\mathrm{Cmac})$ & 1.6, spot 420 \\
\hline MCOT21172.0.CT & Aldehyde dehydrogenase & $0.53(\mathrm{Cmac})$ & 5.4 , spot 626 \\
\hline MCOT23196.0.CT & Myosin heavy chain, isoform $\mathrm{R}$ & $\begin{array}{l}0.17 \text { (Cmac) } \\
0.24 \text { (Cmac HS) }\end{array}$ & 4.1 , spot 626 \\
\hline WP_026092655.1 & Membrane protein [Wolbachia] & $\begin{array}{l}0.89 \text { (Cmac) } \\
1.07 \text { (Cmac HS) }\end{array}$ & 1.9, spot 861 \\
\hline XP_008474229.1 & Uncharacterized protein & $1.69(\mathrm{Cmac})$ & 6.95 , spot 554 \\
\hline
\end{tabular}

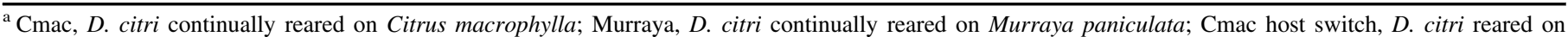
C. macrophylla for 10 days after host switch from M. paniculata; Murraya host switch, D. citri reared on M. paniculata for 10 days after host switch from C. macrophylla; HS, host switch. Shotgun proteomics: the spectral count $\log _{2}$ fold changes between Murraya and the lower abundance sample class under comparison (sample class under comparison in parentheses) are given (Fisher's exact test Benjamini-Hochberg adjusted $P$ value < 0.05). DIGE: the fractional spot volume $\log _{2}$ fold change between Murraya and Murraya host switch is given (threshold of log 2 fold change $>1$ between target and control gel). Gel spot ID in which each protein was identified by DIGE as upregulated in Murraya host switch samples is given.

TABLE 10. Proteins found by both shotgun proteomics and difference gel electrophoresis to be upregulated in Citrus macrophylla-reared Diaphorina citri ${ }^{\text {a }}$

\begin{tabular}{|c|c|c|c|}
\hline Protein ID & Protein description & $\begin{array}{l}\text { Spectral count } \log _{2} \text { fold change (lower } \\
\text { abundance sample under comparison) }\end{array}$ & $\begin{array}{l}\text { Fractional spot volume } \log _{2} \text { fold change } \\
\text { (Cmac versus Murraya HS), spot ID }\end{array}$ \\
\hline MCOT02134.0.CT & Hexamerin-like protein 4 & $\begin{array}{l}2.24 \text { (Murraya HS) } \\
0.93 \text { (Cmac HS) } \\
0.95 \text { (Murraya) }\end{array}$ & $\begin{array}{l}3.19, \text { spot } 146 \\
2.41, \text { spot } 152 \\
8.57, \text { spot } 270 \\
1.61, \text { spot } 272 \\
8.11, \text { spot } 275 \\
8.46, \text { spot } 278 \\
4.71, \text { spot } 301 \\
5.96 \text {, spot } 319 \\
5.1, \text { spot } 342 \\
1.26 \text {, spot } 474 \\
3.48, \text { spot } 580\end{array}$ \\
\hline MCOT17336.0.CT & Arylphorin-like hexamerin-1 & $\begin{array}{l}1.59 \text { (Murraya HS) } \\
0.77 \text { (Murraya) }\end{array}$ & $\begin{array}{l}5.91 \text {, spot } 146 \\
6.02 \text {, spot } 152 \\
3.26 \text {, spot } 272\end{array}$ \\
\hline MCOT21174.0.CT & $\begin{array}{l}\text { DNA-directed RNA polymerase } \\
\text { subunit beta }\end{array}$ & $\begin{array}{l}2.73 \text { (Murraya HS) } \\
1.71 \text { (Murraya) }\end{array}$ & 2.71 , spot 839 \\
\hline XP_008477120.1 & Skin secretory protein $\mathrm{xP} 2$-like & $\begin{array}{l}2.11 \text { (Murraya HS) } \\
1.02 \text { (Cmac HS) } \\
1.21 \text { (Murraya) }\end{array}$ & 4.26 , spot 850 \\
\hline XP_008477908.1 & Hemocyanin 2 & $\begin{array}{l}2.74 \text { (Murraya HS) } \\
1.51 \text { (Murraya) }\end{array}$ & $\begin{array}{l}5.67 \text {, spot } 146 \\
4.6, \text { spot } 152 \\
3.63 \text {, spot } 272 \\
3.6, \text { spot } 557 \\
1.52 \text {, spot } 694 \\
2.08 \text {, spot } 705\end{array}$ \\
\hline XP_008480567.1 & $\begin{array}{l}\text { Uncharacterized protein } \\
\text { LOC } 103517317\end{array}$ & $\begin{array}{l}1.15 \text { (Murraya HS), } \\
3.19 \text { (Murraya) }\end{array}$ & 6.89, spot 835 \\
\hline MCOT13628.0.CT & Arylphorin hexamerin-like protein 2 & 1.23 (Murraya HS) & $\begin{array}{l}3.84, \text { spot } 152 \\
2.85, \text { spot } 272 \\
5.3 \text {, spot } 278 \\
2.56 \text {, spot } 557 \\
3.16 \text {, spot } 705\end{array}$ \\
\hline MCOT15525.0.CO & Pupal cuticle protein $78 \mathrm{E}$ & 3.79 (Murraya HS) & 3.69, spot 557 \\
\hline MCOT12676.2.CT & $\begin{array}{l}\text { Ras GTPase-activating protein- } \\
\text { binding protein } 1\end{array}$ & 1.67 (Murraya) & 6.4, spot 272 \\
\hline
\end{tabular}

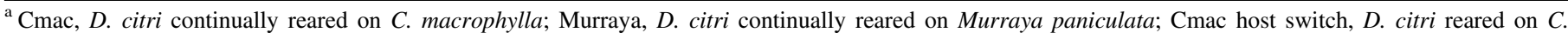
macrophylla for 10 days after host switch from $M$. paniculata; Murraya host switch, D. citri reared on $M$. paniculata for 10 days after host switch from $C$. macrophylla; HS, host switch. Shotgun proteomics: the spectral count $\log _{2}$ fold changes between Cmac and the lower abundance sample class under comparison (sample class under comparison in parentheses) are given (Fisher's exact test Benjamini-Hochberg adjusted $P$ value < 0.05). DIGE: the fractional spot volume $\log _{2}$ fold change between Cmac and Murraya host switch is given (threshold of log fold change $>1$ between target and control gel). Gel spot ID in which each protein was identified by DIGE as upregulated in Cmac samples is given. 


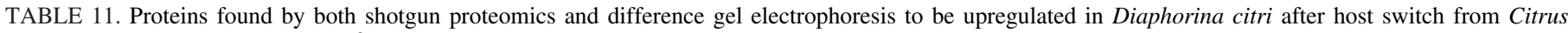
macrophylla to Murraya paniculata ${ }^{\text {a }}$

\begin{tabular}{|c|c|c|c|}
\hline Protein ID & Protein description & $\begin{array}{l}\text { Spectral count } \log _{2} \text { fold change (lower } \\
\text { abundance sample under comparison) }\end{array}$ & $\begin{array}{l}\text { Fractional spot volume } \log _{2} \text { fold change } \\
\text { (Murraya HS versus Cmac), spot ID }\end{array}$ \\
\hline MCOT03242.3.CT & Transferrin & 2.24 (Murraya) & $\begin{array}{l}5.29, \text { spot } 182 \\
8.66 \text {, spot } 197 \\
6.07 \text {, spot } 198\end{array}$ \\
\hline XP_008477906.1 & Hemocyanin 1 & 0.45 (Murraya) & $\begin{array}{l}5.33 \text {, spot } 198 \\
2.47 \text {, spot } 259 \\
4.79 \text {, spot } 197\end{array}$ \\
\hline MCOT03242.2.CO & Transferrin & 1.96 (Murraya) & 3.04 , spot 198 \\
\hline XP_008487106.1 & Vitellogenin-1-like & 1.00 (Murraya) & $\begin{array}{l}6.85 \text {, spot } 867 \\
5.21 \text {, spot } 868 \\
9.64 \text {, spot } 871 \\
4.8 \text {, spot } 872 \\
3.85 \text {, spot } 873 \\
1.63 \text {, spot } 875\end{array}$ \\
\hline MCOT14669.2.CC & Unknown protein & 0.85 (Cmac HS) & 7.4, spot 967 \\
\hline
\end{tabular}

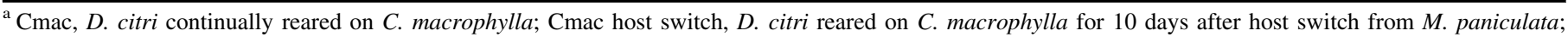
Murraya host switch, D. citri reared on M. paniculata for 10 days after host switch from C. macrophylla; HS, host switch. Shotgun proteomics: the spectral count $\log _{2}$ fold changes between Murraya host switch and the lower abundance sample class under comparison (sample class under comparison in parentheses) are given (Fisher's exact test Benjamini-Hochberg adjusted $P$ value $<0.05$ ). DIGE: the fractional spot volume log ${ }_{2}$ fold change between Murraya host switch and Cmac is given (threshold of $\log _{2}$ fold change $>1$ between target and control gel). Gel spot ID in which each protein was identified by DIGE as upregulated in Murraya host switch samples is given.

$>1 \%$ of genes in insect genomes are typically found to code for cuticle proteins, with distinct expression patterns between tissues and developmental stages (Pan et al. 2018).

A recent study that analyzed transcriptional variation in the generalist aphid herbivore Myzus persicae between insects reared on different host plants identified RR-2 cuticle genes as the family with the highest number of genes upregulated in aphids reared on Nicotiana benthamiana compared with cabbage (Brassica napa) (Mathers et al. 2017). RR-2 cuticle proteins have been identified in a component of the aphid stylet known as the acrostyle, which functions in intracellular probing during phloem feeding (Webster et al. 2017). The differential expression of RR-2 cuticle genes during host plant adaptation suggests that variation in cuticle composition enables $M$. persicae to adjust to physical and chemical differences between plant hosts (Mathers et al. 2017). The finding that RR-2 cuticle proteins in $D$. citri are differentially expressed between insects adapted to different host plants, taken together with this previous discovery of transcriptional changes in $M$. persicae cuticle genes, suggests that variation in cuticle gene expression may be an important component of host plant adaptation in hemipteran insects.

The identification of proteins differentially expressed between $D$. citri samples by both DIGE and shotgun proteomics provides a high degree of confidence that these represent significant differences because of their discovery by two separate experimental workflows. However, there were cases in which proteins with biologically compelling functions were identified in only one of the two platforms, which is expected given the differences between what each experimental workflow is analyzing. These cases illustrate the complementary power of these two approaches because there are situations in which either DIGE or shotgun proteomics is more likely to identify a given protein. The most abundant protein in one of the Murraya host switch-upregulated DIGE spots is a protein annotated as propionyl-CoA-carboxylase, while this protein was not identified at all by shotgun proteomics. This protein functions in valine catabolism and is present in most animals, but the gene for this protein has not been found in most insects with fully sequenced genomes. Propionyl-CoA-carboxylase was found at low abundance in previous $D$. citri shotgun proteomics analysis, with significantly more spectral counts in CLas-exposed insects compared with those collected from healthy citrus (Ramsey et al. 2015). In this study, the protein was likely present at too low abundance to be detected by shotgun proteomics, but protein sample fractionation by 2-D gel electrophoresis provided the resolution to identify a gel spot with significantly different fluorescence intensity between samples which contained this protein. In contrast, four different aminopeptidase $\mathrm{N}$ proteins were identified by shotgun proteomics as upregulated in Murraya samples (Supplementary Table S7), whereas only one of these proteins was identified by DIGE as a minor component of a gel spot that was not found to be differentially expressed. Aminopeptidase $\mathrm{N}$ has been identified in the pea aphid (Acyrthosiphon pisum) gut as the receptor for Pea enation mosaic virus (Linz et al. 2015) and functions in the cotton bollworm (Helicoverpa armigera) by binding to toxins produced by the Bacillus thuringiensis (Zhao et al. 2017). Possible reasons the Aminopeptidase N may not have been identified as differentially expressed by DIGE include comigration with other nondifferentially expressed proteins. These results reveal the power of combining top-down and bottom-up proteomics for the identification of differentially expressed proteins between different sample classes.

\section{LITERATURE CITED}

Alves, M. N., Cifuentes-Arenas, J. C., Raiol-Junior, L. L., Ferro, J. A., and Pena, L. 2021. Early population dynamics of "Candidatus Liberibacter asiaticus" in susceptible and resistant genotypes after inoculation with infected Diaphorina citri feeding on young shoots. Front. Microbiol. 12:683923.

Ammar, E., Walter, A. J., and Hall, D. G. 2013. New excised-leaf assay method to test inoculativity of Asian citrus psyllid (Hemiptera: Psyllidae) with Candidatus Liberibacter asiaticus associated with citrus huanglongbing disease. J. Econ. Entomol. 106:25-35.

Ammar, E. D., Hall, D. G., Hosseinzadeh, S., and Heck, M. 2018. The quest for a non-vector psyllid: Natural variation in acquisition and transmission of the huanglongbing pathogen 'Candidatus Liberibacter asiaticus' by Asian citrus psyllid isofemale lines. PLoS One 13:e0195804.

Aranda, A., Maugeri, D., Uttaro, A. D., Opperdoes, F., Cazzulo, J. J., and Nowicki, C. 2006. The malate dehydrogenase isoforms from Trypanosoma brucei: Subcellular localization and differential expression in bloodstream and procyclic forms. Int. J. Parasitol. 36:295-307.

Beerntsen, B. T., James, A. A., and Christensen, B. M. 2000. Genetics of mosquito vector competence. Microbiol. Mol. Biol. Rev. 64:115-137.

Beloti, V. H., Alves, G. R., Coletta-Filho, H. D., and Yamamoto, P. T. 2018. The Asian citrus psyllid host Murraya koenigii is immune to citrus huanglongbing pathogen 'Candidatus Liberibacter asiaticus'. Phytopathology 108:1089-1094.

Burmester, T. 2002. Origin and evolution of arthropod hemocyanins and related proteins. J. Comp. Physiol. B 172:95-107.

Burmester, T. 2015. Expression and evolution of hexamerins from the tobacco hornworm, Manduca sexta, and other Lepidoptera. Insect Biochem. Mol. Biol. 62:226-234.

Chu, C. C., Gill, T. A., Hoffmann, M., and Pelz-Stelinski, K. S. 2016. Interpopulation variability of endosymbiont densities in the Asian citrus psyllid (Diaphorina citri Kuwayama). Microb. Ecol. 71:999-1007. 
Cifuentes-Arenas, J. C., Beattie, G. A. C., Pena, L., and Lopes, S. A. 2019. Murraya paniculata and Swinglea glutinosa as short-term transient hosts of 'Candidatus Liberibacter asiaticus' and implications for the spread of huanglongbing. Phytopathology 109:2064-2073.

Cilia, M., Fish, T., Yang, X., McLaughlin, M., Thannhauser, T. W., and Gray, S. 2009. A comparison of protein extraction methods suitable for gel-based proteomic studies of aphid proteins. J. Biomol. Tech. 20:201-215.

Cilia, M., Howe, K., Fish, T., Smith, D., Mahoney, J., Tamborindeguy, C., Burd, J., Thannhauser, T. W., and Gray, S. 2011b. Biomarker discovery from the top down: Protein biomarkers for efficient virus transmission by insects (Homoptera: Aphididae) discovered by coupling genetics and 2-D DIGE. Proteomics 11:2440-2458.

Cilia, M., Tamborindeguy, C., Rolland, M., Howe, K., Thannhauser, T. W., and Gray, S. 2011a. Tangible benefits of the aphid Acyrthosiphon pisum genome sequencing for aphid proteomics: Enhancements in protein identification and data validation for homology-based proteomics. J. Insect Physiol. 57:179-190.

Damsteegt, V. D., Postnikova, E. N., Stone, A. L., Kuhlmann, M., Wilson, C., Sechler, A., Schaad, N. W., Brlansky, R. H., and Schneider, W. L. 2010. Murraya paniculata and related species as potential hosts and inoculum reservoirs of 'Candidatus Liberibacter asiaticus', causal agent of huanglongbing. Plant Dis. 94:528-533.

Decker, H., and Jaenicke, E. 2004. Recent findings on phenoloxidase activity and antimicrobial activity of hemocyanins. Dev. Comp. Immunol. 28:673-687.

Erban, T., Jedelsky, P. L., and Titera, D. 2013. Two-dimensional proteomic analysis of honeybee, Apis mellifera, winter worker hemolymph. Apidologie (Celle) 44:404-418.

George, J., and Lapointe, S. L. 2018. Host plant resistance associated with Poncirus trifoliata influence oviposition, development and adult emergence of Diaphorina citri (Hemiptera: Liviidae). Pest Manag. Sci. 75:279-285.

Hall, D. G., George, J., and Lapointe, S. L. 2015. Further investigations on colonization of Poncirus trifoliata by the Asian citrus psyllid. Crop Prot. 72:112-118.

Hall, D. G., and Hentz, M. G. 2016. An evaluation of plant genotypes for rearing Asian citrus psyllid (Hemiptera: Liviidae). Fla. Entomol. 99:471-480.

Hall, D. G., and Moulton, K. M. 2018. Transmission rates of 'Candidatus Liberibacter asiaticus' to greenhouse seedlings by laboratory colonies of Asian citrus psyllid (Hemiptera: Liviidae). J. Econ. Entomol. 111:2546-2552.

Hall, D. G., Richardson, M. L., Ammar, E. D., and Halbert, S. E. 2013. Asian citrus psyllid, Diaphorina citri, vector of citrus huanglongbing disease. Entomol. Exp. Appl. 146:207-223.

Heck, M. 2018. Insect transmission of plant pathogens: A systems biology perspective. mSystems 3:3.

Hedrick, T. L., Combes, S. A., and Miller, L. A. 2015. Recent developments in the study of insect flight. Can. J. Zool. 93:925-943.

Hodge, K., Ten Have, S., Hutton, L., and Lamond, A. I. 2013. Cleaning up the masses: Exclusion lists to reduce contamination with HPLC-MS/MS. J. Proteomics 88:92-103.

Ishihama, Y., Oda, Y., Tabata, T., Sato, T., Nagasu, T., Rappsilber, J., and Mann, M. 2005. Exponentially modified protein abundance index (emPAI) for estimation of absolute protein amount in proteomics by the number of sequenced peptides per protein. Mol. Cell. Proteomics 4:1265-1272.

Killiny, N. 2022. Made for each other: Vector-pathogen interfaces in the huanglongbing pathosystem. Phytopathology 112:26-43.

Kruse, A., Ramsey, J. S., Johnson, R., Hall, D. G., MacCoss, M. J., and Heck, M. 2018. 'Candidatus Liberibacter asiaticus' minimally alters expression of immunity and metabolism proteins in the hemolymph of Diaphorina citri, the insect vector of Huanglongbing. J. Proteome Res. 17:2995-3011.

Lee, J. A., Halbert, S. E., Dawson, W. O., Robertson, C. J., Keesling, J. E., and Singer, B. H. 2015. Asymptomatic spread of huanglongbing and implications for disease control. Proc. Natl. Acad. Sci. USA 112:7605-7610.

Lee, S. Y., Lee, B. L., and Soderhall, K. 2003. Processing of an antibacterial peptide from hemocyanin of the freshwater crayfish Pacifastacus leniusculus. J. Biol. Chem. 278:7927-7933.

Linz, L. B., Liu, S., Chougule, N. P., and Bonning, B. C. 2015. In vitro evidence supports membrane alanyl aminopeptidase $\mathrm{N}$ as a receptor for a plant virus in the pea aphid vector. J. Virol. 89:11203-11212.

Martini, X., Hoyte, A., and Stelinski, L. L. 2014. Abdominal color of the Asian citrus psyllid (Hemiptera: Liviidae) is associated with flight capabilities. Ann. Entomol. Soc. Am. 107:842-847.

Mathers, T. C., Chen, Y. Z., Kaithakottil, G., Legeai, F., Mugford, S. T., Baa-Puyoulet, P., Bretaudeau, A., Clavijo, B., Colella, S., Collin, O., Dalmay, T., Derrien, T., Feng, H. L., Gabaldon, T., Jordan, A., Julca, I., Kettles, G. J., Kowitwanich, K., Lavenier, D., Lenzi, P., Lopez-Gomollon, S., Loska, D., Mapleson, D., Maumus, F., Moxon, S., Price, D. R. G., Sugio, A., van Munster, M., Uzest, M., Waite, D., Jander, G., Tagu, D., Wilson, A. C. C., van Oosterhout, C., Swarbreck, D., and Hogenhout, S. A. 2017. Rapid transcriptional plasticity of duplicated gene clusters enables a clonally reproducing aphid to colonize diverse plant species. Genome Biol. 18:27.
McCollum, G., Hilf, M., Irey, M., Luo, W. Q., and Gottwald, T. 2016. Susceptibility of sixteen citrus genotypes to 'Candidatus Liberibacter asiaticus'. Plant Dis. 100:1080-1086.

Mellroth, P., and Steiner, H. 2006. PGRP-SB1: An N-acetylmuramoyl L-alanine amidase with antibacterial activity. Biochem. Biophys. Res. Commun. 350:994-999.

Pan, P. L., Ye, Y. X., Lou, Y. H., Lu, J. B., Cheng, C., Shen, Y., Moussian, B., and Zhang, C. X. 2018. A comprehensive omics analysis and functional survey of cuticular proteins in the brown planthopper. Proc. Natl. Acad. Sci. USA 115:5175-5180.

Paris, T. M., Allan, S. A., Hall, D. G., Hentz, M. G., Hetesy, G., and Stansly, P. A. 2016. Host plant affects morphometric variation of Diaphorina citri (Hemiptera: Liviidae). PeerJ 4:e2450v1.

Pinheiro, P. V., Ghanim, M., Alexander, M., Rebelo, A. R., Santos, R. S., Orsburn, B. C., Gray, S., and Cilia, M. 2017. Host plants indirectly influence plant virus transmission by altering gut cysteine protease activity of aphid vectors. Mol. Cell. Proteomics 16:S230-S243.

Ramadugu, C., Keremane, M. L., Halbert, S. E., Duan, Y. P., Roose, M. L., Stover, E., and Lee, R. F. 2016. Long-term field evaluation reveals huanglongbing resistance in citrus relatives. Plant Dis. 100:1858-1869.

Ramsey, J. S., Chavez, J. D., Johnson, R., Hosseinzadeh, S., Mahoney, J. E., Mohr, J. P., Robison, F., Zhong, X., Hall, D. G., MacCoss, M., Bruce, J., and Cilia, M. 2017. Protein interaction networks at the host-microbe interface in Diaphorina citri, the insect vector of the citrus greening pathogen. R. Soc. Open Sci. 4:160545.

Ramsey, J. S., Johnson, R. S., Hoki, J. S., Kruse, A., Mahoney, J., Hilf, M. E., Hunter, W. B., Hall, D. G., Schroeder, F. C., MacCoss, M. J., and Cilia, M. 2015. Metabolic interplay between the Asian citrus psyllid and its profftella symbiont: An Achilles' heel of the citrus greening insect vector. PLoS One 10: e0140826.

Rebers, J. E., and Riddiford, L. M. 1988. Structure and Expression of a Manduca sexta larval cuticle gene homologous to Drosophila cuticle genes. J. Mol. Biol. 203:411-423.

Rupprecht, E., Gathmann, S., Fuhrmann, E., and Schneider, D. 2007. Three different DnaK proteins are functionally expressed in the cyanobacterium Synechocystis sp. PCC 6803. Microbiol-Sgm 153:1828-1841.

Saha, S., Hosmani, P. S., Villalobos-Ayala, K., Miller, S., Shippy, T., Flores, M., Rosendale, A., Cordola, C., Bell, T., Mann, H., DeAvila, G., DeAvila, D., Moore, Z., Buller, K., Ciolkevich, K., Nandyal, S., Mahoney, R., Van Voorhis, J., Dunlevy, M., Farrow, D., Hunter, D., Morgan, T., Shore, K., Guzman, V., Izsak, A., Dixon, D. E., Cridge, A., Cano, L., Cao, X., Jiang, H., Leng, N., Johnson, S., Cantarel, B. L., Richards, S., English, A., Shatters, R. G., Childers, C., Chen, M. J., Hunter, W., Cilia, M., Mueller, L. A., Munoz-Torres, M., Nelson, D., Poelchau, M. F., Benoit, J. B., Wiersma-Koch, H., D'Elia, T., and Brown, S. J. 2017. Improved annotation of the insect vector of citrus greening disease: Biocuration by a diverse genomics community. Database (Oxford) 2017:2017.

Salmela, H., Amdam, G. V., and Freitak, D. 2015. Transfer of immunity from mother to offspring is mediated via egg-yolk protein vitellogenin. PLoS Pathog 11: e1005015.

Smith, L. M., and Kelleher, N. L. 2018. Proteoforms as the next proteomics currency. Science 359:1106-1107.

Smith, L. M., Kelleher, N. L., and Proteomics, C. T. D. 2013. Proteoform: A single term describing protein complexity. Nat. Methods 10:186-187.

Ueda, N., and Boettcher, A. 2009. Differences in heat shock protein 70 expression during larval and early spat development in the Eastern oyster, Crassostrea virginica (Gmelin, 1791). Cell Stress Chaperones 14:439-443.

Vizcaino, J. A., Csordas, A., del-Toro, N., Dianes, J. A., Griss, J., Lavidas, I., Mayer, G., Perez-Riverol, Y., Reisinger, F., Ternent, T., Xu, Q. W., Wang, R., and Hermjakob, H. 2016. 2016 update of the PRIDE database and its related tools. Nucleic Acids Res. 44:D447-D456.

Vizcaino, J. A., Deutsch, E. W., Wang, R., Csordas, A., Reisinger, F., Rios, D., Dianes, J. A., Sun, Z., Farrah, T., Bandeira, N., Binz, P. A., Xenarios, I., Eisenacher, M., Mayer, G., Gatto, L., Campos, A., Chalkley, R. J., Kraus, H. J., Albar, J. P., Martinez-Bartolome, S., Apweiler, R., Omenn, G. S., Martens, L., Jones, A. R., and Hermjakob, H. 2014. ProteomeXchange provides globally coordinated proteomics data submission and dissemination. Nat. Biotechnol. 32:223-226.

Wagner, C. R., Mahowald, A. P., and Miller, K. G. 2002. One of the two cytoplasmic actin isoforms in Drosophila is essential. Proc. Natl. Acad. Sci. USA 99:8037-8042.

Walter, A. J., Duan, Y. P., and Hall, D. G. 2012b. Titers of 'Ca. Liberibacter asiaticus' in Murraya paniculata and Murraya-reared Diaphorina citri are much lower than in citrus and citrus-reared psyllids. HortScience 47:14491452 .

Walter, A. J., Hall, D. G., and Duan, Y. P. 2012a. Low incidence of 'Candidatus Liberibacter asiaticus' in Murraya paniculata and associated Diaphorina citri. Plant Dis. 96:827-832. 
Webster, C. G., Thillier, M., Pirolles, E., Cayrol, B., Blanc, S., and Uzest, M. 2017. Proteomic composition of the acrostyle: Novel approaches to identify cuticular proteins involved in virus-insect interactions. Insect Sci. 24:990-1002.

Willis, J. H. 2010. Structural cuticular proteins from arthropods: Annotation, nomenclature, and sequence characteristics in the genomics era. Insect Biochem. Mol. Biol. 40:189-204.

Yang, Y., Thannhauser, T. W., Li, L., and Zhang, S. 2007. Development of an integrated approach for evaluation of 2-D gel image analysis: Impact of multiple proteins in single spots on comparative proteomics in conventional 2-D gel/MALDI workflow. Electrophoresis 28:2080-2094.
Zhao, M., Yuan, X. D., Wei, J. Z., Zhang, W. N., Wang, B. J., Khaing, M. M., and Liang, G. M. 2017. Functional roles of cadherin, aminopeptidase- $\mathrm{N}$ and alkaline phosphatase from Helicoverpa armigera (Hubner) in the action mechanism of Bacillus thuringiensis Cry2Aa. Sci Rep-Uk 7.

Zheng, Y., Sweet, S. M., Popovic, R., Martinez-Garcia, E., Tipton, J. D., Thomas, P. M., Licht, J. D., and Kelleher, N. L. 2012. Total kinetic analysis reveals how combinatorial methylation patterns are established on lysines 27 and 36 of histone H3. Proc. Natl. Acad. Sci. USA 109:1354913554. 